\title{
Soliton interaction with small Toeplitz potentials for the Szegö equation on $\mathbb{R}$
}

\author{
Oana Pocovnicu \\ Communicated by Yvan Martel, received August 8, 2011.
}

\begin{abstract}
We consider the cubic Szegö equation with a small Toeplitz potential and with soliton initial data$$
\left\{\begin{array}{l}
i \partial_{t} u=\Pi\left(|u|^{2} u\right)+\varepsilon T_{b} u \\
u(0, x)=\alpha_{0} e^{i \phi_{0}} \mu_{0} \eta\left(\mu_{0}\left(x-a_{0}\right)\right) .
\end{array}\right.
$$

We show that up to time $\varepsilon^{-1 / 2} \log (1 / \varepsilon)$ and errors of size $\varepsilon^{1 / 2}$, the solution preserves the soliton shape $u(t, x)=\alpha e^{i \phi} \mu \eta(\mu(x-a))$, and the time dependent parameters $a, \alpha, \phi, \mu$ evolve according to the effective dynamics, up to small corrections.
\end{abstract}

\section{Contents}

1. Introduction

2. Manifold of solitons

3. Effective dynamics 8

4. Reparametrized evolution 10

5. Coerciveness of the linearized operator $\mathcal{L} \quad 18$

6. Main estimates 19

7. Proof of Theorem $1.1 \quad 25$

References $\quad 27$

\section{Introduction}

One of the most important properties in the study of the nonlinear Schrödinger equations (NLS) is dispersion. It is often exhibited in the form of the Strichartz estimates of the corresponding linear flow. In case of the cubic NLS:

$$
i \partial_{t} u+\Delta u=|u|^{2} u, \quad(t, x) \in \mathbb{R} \times M,
$$

2000 Mathematics Subject Classification. 35B15, 35Q51, 37K40, 37K10, 47B35.

Key words and phrases. Szegö equation; soliton; effective Hamiltonian; Toeplitz operators. 
Gérard and Grellier [6] remarked that there is a lack of dispersion when $M$ is a sub-Riemannian manifold (for example, the Heisenberg group). In this situation, many of the classical arguments used in the study of NLS no longer hold. As a consequence, even the problem of global well-posedness of (1.1) on a sub-Riemannian manifold still remains open. In [5, 6], Gérard and Grellier introduced a model of a non-dispersive Hamiltonian equation called the cubic Szëgo equation. (See (1.2) below.) The study of this equation is expected to give new tools to be used in understanding existence and other properties of smooth solutions of NLS in the absence of dispersion.

In this paper we will consider the Szegö equation on the real line. The space of solutions in this case is the Hardy space $L_{+}^{2}(\mathbb{R})$ on the upper half-plane $\mathbb{C}_{+}=\{z ; \operatorname{Im} z>0\}$, defined by

$$
L_{+}^{2}(\mathbb{R})=\left\{f \in L^{2}(\mathbb{R}) ; \operatorname{supp} \hat{f} \subset[0, \infty)\right\} .
$$

The corresponding Sobolev spaces $H_{+}^{s}(\mathbb{R}), s \geq 0$ are defined by:

$$
H_{+}^{s}(\mathbb{R})=\left\{h \in L_{+}^{2}(\mathbb{R}) ;\|h\|_{H_{+}^{s}}:=\left(\frac{1}{2 \pi} \int_{0}^{\infty}\left(1+|\xi|^{2}\right)^{s}|\hat{h}(\xi)|^{2} d \xi\right)^{1 / 2}<\infty\right\} .
$$

The Szegö projector $\Pi$ is the projector on the non-negative frequencies, $\Pi: L^{2}(\mathbb{R}) \rightarrow L_{+}^{2}(\mathbb{R})$

$$
\Pi(f)(x)=\frac{1}{2 \pi} \int_{0}^{\infty} e^{i x \xi} \hat{f}(\xi) d \xi .
$$

For $u \in L_{+}^{2}(\mathbb{R})$, we consider the Szëgo equation on the real line:

$$
i \partial_{t} u=\Pi\left(|u|^{2} u\right), \quad(t, x) \in \mathbb{R} \times \mathbb{R} .
$$

This equation is globally well-posed in $H_{+}^{\frac{1}{2}}(\mathbb{R})$.

On $L_{+}^{2}(\mathbb{R})$ we introduce the symplectic form

$$
\omega(u, v)=\operatorname{Im} \int_{\mathbb{R}} u \bar{v} d x
$$

and the real scalar product

$$
\langle u, v\rangle=\operatorname{Re} \int_{\mathbb{R}} u \bar{v} d x .
$$

Let $\mathcal{D} \subset L_{+}^{2}(\mathbb{R})$ be a dense subset of $L_{+}^{2}(\mathbb{R})$. We say that a function $F: \mathcal{D} \rightarrow \mathbb{R}$ admits a Hamiltonian vector field $X_{F}: \mathcal{D} \rightarrow L_{+}^{2}(\mathbb{R})$ if

$$
d_{u} F(h)=\omega\left(h, X_{F}(u)\right),
$$

for all $u, h \in \mathcal{D}$. The function

$$
H(u)=\frac{1}{4} \int_{\mathbb{R}}|u(x)|^{4} d x
$$

defined on $L_{+}^{4}(\mathbb{R})$, admits the Hamiltonian vector field

$$
X_{H}(u)=-i \Pi\left(|u|^{2} u\right)
$$

Thus the Szegö equation is a Hamiltonian evolution. The most remarkable property of this equation is the fact that it is completely integrable in the sense that it posses a Lax pair structure [13]. The Lax pair is given in terms of Hankel and Toeplitz operators. 
A Hankel operator $H_{u}: L_{+}^{2} \rightarrow L_{+}^{2}$ of symbol $u \in H_{+}^{1 / 2}$ is defined by

$$
H_{u}(h)=\Pi(u \bar{h}) \text {. }
$$

$H_{u}$ is a Hilbert-Schmidt operator, it is $\mathbb{C}$-anti-linear and satisfies

$$
\left(H_{u}\left(h_{1}\right), h_{2}\right)=\left(H_{u}\left(h_{2}\right), h_{1}\right) .
$$

A Toeplitz operator $T_{b}: L_{+}^{2} \rightarrow L_{+}^{2}$ of symbol $b \in L^{\infty}(\mathbb{R})$ is defined by

$$
T_{b}(h)=\Pi(b h) .
$$

$T_{b}$ is $\mathbb{C}$-linear and bounded. Moreover, $T_{b}$ is self-adjoint if and only if $b$ is realvalued.

In what follows we consider the perturbed Szegö equation with a small Toeplitz potential

$$
i \partial_{t} u=\Pi\left(|u|^{2} u\right)+\varepsilon T_{b} u .
$$

This is no longer a completely integrable equation. It is still globally well posed in $H_{+}^{\frac{1}{2}}(\mathbb{R})$ if $b \in H^{1}(\mathbb{R})$. This can be proved by following the lines of the proof of Theorem 2.1 in [5] on the global well-posedness of the Szegö equation.

If instead of the Toeplitz potential we considered a multiplicative linear potential $b u$, then the corresponding equation would no longer be Hamiltonian. However, if we project to $L_{+}^{2}$, obtaining this way a Toeplitz potential $T_{b} u=\Pi(b u)$, we conserve the Hamiltonian structure of the Szegö equation. For this reason, the Toeplitz potential is the natural generalization of the linear multiplicative potential in the case of the Szegö equation.

The Hamiltonian of equation (1.4) is

$$
H_{b}(u)=\frac{1}{4} \int_{\mathbb{R}}|u(x)|^{4} d x+\frac{\varepsilon}{2} \int_{\mathbb{R}} b(x)|u(x)|^{2} d x .
$$

This yields that the Hamiltonian $H_{b}$ is formally conserved by the flow. Note also that the fact that $b$ is a real valued function, yields the conservation of the mass $Q(u)=\int|u|^{2} d x$.

The goal of the paper is to study the long time behavior of the solution of the perturbed Szegö equation (1.4) having as initial condition a soliton of the unperturbed equation.

Definition 1. A soliton for the Szegö equation on the real line is a solution $u$ with the property that there exist $c, \omega \in \mathbb{R}, c \neq 0$ such that

$$
u(t, x)=e^{-i t \omega} u_{0}(x-c t) .
$$

In [13, Theorem 2] it was proved that all the initial data of solitons for the Szegö equation on $\mathbb{R}$ are of the form

$$
u_{0}=e^{i \phi_{0}} \alpha_{0} \mu_{0} \eta\left(\mu_{0}\left(x-a_{0}\right)\right)=\frac{e^{i \phi_{0}} \alpha_{0}}{x-a_{0}+\frac{i}{\mu_{0}}},
$$

where $\eta(x):=\frac{1}{x+i}, \alpha_{0}, \mu_{0} \in(0, \infty)$, and $\phi_{0}, a_{0} \in \mathbb{R}$, and that the corresponding solution is

$$
u(t, x)=e^{i \phi(t)} \alpha_{0} \mu_{0} \eta\left(\mu_{0}(x-a(t))\right)=\frac{e^{i \phi(t)} \alpha_{0}}{x-a(t)+\frac{i}{\mu_{0}}},
$$

where $\phi(t)=-\frac{\alpha_{0}^{2} \mu_{0}^{2}}{4} t+\phi_{0}$ and $a(t)=\frac{\alpha_{0}^{2} \mu_{0}}{2} t+a_{0}$. 
We show that the solution of the perturbed Szegö equation (1.4) with initial data $u_{0}=e^{i \phi_{0}} \alpha_{0} \mu_{0} \eta\left(\mu_{0}\left(x-a_{0}\right)\right)$ preserves the form $u=e^{i \phi} \alpha \mu \eta(\mu(x-a))$ over a large interval of time, and the time dependent parameters $a, \alpha, \phi, \mu$ evolve according to the effective dynamics, up to small corrections. More precisely, the main result of the paper is the following theorem.

THEOREM 1.1. Let $b: \mathbb{R} \rightarrow \mathbb{R}$ be a function in $H^{1}(\mathbb{R})$ with the property that $b^{\prime} \in L^{1}(\mathbb{R})$. Let $0<\varepsilon \ll 1$ and $0<\delta<\frac{1}{2}$. If $u$ is a solution of the perturbed Szegö equation with a small Toeplitz potential

$$
\left\{\begin{array}{l}
i \partial_{t} u=\Pi\left(|u|^{2} u\right)+\varepsilon T_{b} u \\
u(0, x)=\alpha_{0} e^{i \phi_{0}} \mu_{0} \eta\left(\mu_{0}\left(x-a_{0}\right)\right),
\end{array}\right.
$$

where $a_{0}, \phi_{0} \in \mathbb{R}$ and $\alpha_{0}, \mu_{0} \in(0, \infty)$, then

$$
\left\|u(t)-\alpha(t) e^{i \phi(t)} \mu(t) \eta(\mu(t)(x-a(t)))\right\|_{H_{+}^{\frac{1}{2}}} \leq C \varepsilon^{\frac{1}{2}+\frac{\delta}{3}},
$$

for times $0 \leq t \leq \frac{\delta}{6 \ln c_{0}} \cdot \frac{1}{\varepsilon^{\frac{1}{2}-\delta}} \ln \left(\frac{1}{\varepsilon}\right)$, where $c_{0}$ is a constant depending only on $\alpha_{0}$ and $\mu_{0}$, and $a, \alpha, \phi, \mu$ satisfy

$$
\left\{\begin{array}{l}
\dot{a}=\frac{\alpha^{2} \mu}{2}-\frac{2 \varepsilon}{\pi \mu} \int b^{\prime}\left(a+\frac{x}{\mu}\right) \frac{x}{\mu}|\eta(x)|^{2} d x+O\left(\varepsilon^{1+\frac{2 \delta}{3}}\right), \\
\dot{\alpha}=\frac{\varepsilon \alpha}{\pi \mu} \int b^{\prime}\left(a+\frac{x}{\mu}\right)|\eta(x)|^{2} d x+O\left(\varepsilon^{1+\frac{2 \delta}{3}}\right), \\
\dot{\phi}=-\frac{\alpha^{2} \mu^{2}}{4}-\frac{\varepsilon}{\pi} \int b\left(a+\frac{x}{\mu}\right)|\eta(x)|^{2} d x-\frac{\varepsilon}{\pi} \int b^{\prime}\left(a+\frac{x}{\mu}\right) \frac{x}{\mu}|\eta(x)|^{2} d x+O\left(\varepsilon^{1+\frac{2 \delta}{3}}\right), \\
\dot{\mu}=-\frac{2 \varepsilon}{\pi} \int b^{\prime}\left(a+\frac{x}{\mu}\right)|\eta(x)|^{2} d x+O\left(\varepsilon^{1+\frac{2 \delta}{3}}\right) .
\end{array}\right.
$$

In addition, if $\bar{a}, \bar{\alpha}, \bar{\phi}, \bar{\mu}$ satisfy

$$
\left\{\begin{array}{l}
\dot{\bar{a}}=\frac{\bar{\alpha}^{2} \bar{\mu}}{2}-\frac{2 \varepsilon}{\pi \bar{\mu}} \int b^{\prime}\left(\bar{a}+\frac{x}{\bar{\mu}}\right) \frac{x}{\bar{\mu}}|\eta(x)|^{2} d x \\
\dot{\bar{\alpha}}=\frac{\varepsilon \bar{\alpha}}{\pi \bar{\mu}} \int b^{\prime}\left(\bar{a}+\frac{x}{\bar{\mu}}\right)|\eta(x)|^{2} d x \\
\dot{\bar{\phi}}=-\frac{\bar{\alpha}^{2} \bar{\mu}^{2}}{4}-\frac{\varepsilon}{\pi} \int b\left(\bar{a}+\frac{x}{\bar{\mu}}\right)|\eta(x)|^{2} d x-\frac{\varepsilon}{\pi} \int b^{\prime}\left(\bar{a}+\frac{x}{\bar{\mu}}\right) \frac{x}{\bar{\mu}}|\eta(x)|^{2} d x \\
\dot{\bar{\mu}}=-\frac{2 \varepsilon}{\pi} \int b^{\prime}\left(\bar{a}+\frac{x}{\bar{\mu}}\right)|\eta(x)|^{2} d x
\end{array}\right.
$$

with the same initial data $a_{0}, \alpha_{0}, \phi_{0}, \mu_{0}$, then

$$
\left\{\begin{array}{l}
|a-\bar{a}| \leq \tilde{c}_{0} \delta \varepsilon^{\frac{1}{2}+\delta} \ln \left(\frac{1}{\varepsilon}\right), \\
|\alpha-\bar{\alpha}| \leq \tilde{c}_{0} \delta \varepsilon^{\frac{1}{2}+\delta} \ln \left(\frac{1}{\varepsilon}\right), \\
|\phi-\bar{\phi}| \leq \tilde{c}_{0} \delta \varepsilon^{2 \delta} \ln \left(\frac{1}{\varepsilon}\right)^{2} \\
|\mu-\bar{\mu}| \leq \tilde{c}_{0} \delta \varepsilon^{\frac{1}{2}+\delta} \ln \left(\frac{1}{\varepsilon}\right) .
\end{array}\right.
$$

where $\tilde{c}_{0}$ depends on $\alpha_{0}, \mu_{0}$.

As a consequence, if $\varepsilon$ is small enough and $\frac{3}{10}<\delta<\frac{1}{2}$, then for times $0 \leq t \leq \frac{\delta}{6 \ln c_{0}} \cdot \frac{1}{\varepsilon^{\frac{1}{2}-\delta}} \ln \left(\frac{1}{\varepsilon}\right)$ we have that

$$
\left\|u(t)-\bar{\alpha}(t) e^{i \bar{\phi}(t)} \bar{\mu}(t) \eta(\bar{\mu}(t)(x-\bar{a}(t)))\right\|_{H_{+}^{\frac{1}{2}}} \leq C \varepsilon^{\frac{1}{2}+\frac{\delta}{3}} .
$$

The problem of studying the solution of a perturbed equation having as initial condition a soliton of the unperturbed equation was first addressed in the setting of the nonlinear Schrödinger equation by Bronski and Jerrard in [1] and their result was improved by Keraani in $[\mathbf{1 0}, \mathbf{1 1}]$. They considered the semiclassical regime which is equivalent to adding a slowly varying potential $V(\varepsilon x)$. The method consists 
in using the orbital stability of the soliton and the result states that the center of mass moves according to Newton's equation $a^{\prime \prime}(t)=-D V(a)$. It seems difficult to adapt this method to the setting of the Szegö equation since it extensively exploits the relations between the densities of mass, energy, and momentum. These identities have no correspondent for the Szegö equation.

This problem was also considered by Fröhlich, Tsai, and Yau and Fröhlich, Gustafson, Jonsson, and Sigal in the settings of the Hartree equation and of the nonlinear Schrödinger equation with a general nonlinearity in $[4,2,3]$. Some of these results were improved in $[\mathbf{7}, \mathbf{8}]$ by Zworski and Holmer in the case of the one dimensional nonlinear Schrödinger equation with a Dirac potential and with a slowly varying potential. In this paper we adapt the method of Zworski and Holmer to the case of the Szegö equation.

The starting point in proving Theorem 1.1 is to determine the vector field corresponding to the restriction $\left.H_{b}\right|_{M}$ of the Hamiltonian to the four-dimensional manifold of solitons

$$
\left.M=\left\{e^{i \phi} \alpha \mu \eta(x-a)\right), \phi, a \in \mathbb{R}, \alpha>0, \mu>0\right\} .
$$

Then, we determine the flow of this vector field, called the effective dynamics. In the case of the Szegö equation with a small Toeplitz potential the effective dynamics are given in the system (1.9). We then decompose the flow of the perturbed Szegö equation (1.4) into a part belonging to the manifold $M$ and a part which is symplectically orthogonal to $M$. We show that the part of the solution which is orthogonal to $M$ is small. Thus, the flow of (1.4) is close to $M$. Then, the heuristics pointed out by Holmer and Zworski suggest that the flow is close to the flow of $\left.H_{b}\right|_{M}$, i.e. the effective dynamics. This can be rigorously proved and yields the approximation (1.11).

In proving that the part of the flow which is orthogonal to $M$ is small we consider the Lyapunov functional and use the coerciveness of the linearized operator. First we consider the functional $\mathcal{E}: H_{+}^{1 / 2} \rightarrow \mathbb{R}$,

$$
\mathcal{E}(u)=\frac{1}{4} \int|u|^{4} d x+\frac{i}{4} \int\left(\partial_{x} u\right) \bar{u} d x-\frac{1}{8} \int|u|^{2} d x .
$$

Then $\eta=\frac{1}{x+i}$ is a critical point of $\mathcal{E}$, i.e. $d_{\eta} \mathcal{E}=0$ since

$$
\frac{i}{2} \partial_{x} \eta+\Pi\left(|\eta|^{2} \eta\right)-\frac{\eta}{4}=0 .
$$

The Lyapunov functional is defined by

$$
L(w)=\mathcal{E}(w+\eta)-\mathcal{E}(w)
$$

and the linearized operator $\mathcal{L}: H_{+}^{\frac{1}{2}} \rightarrow \mathbb{R}$ is

$$
\mathcal{L}(w)=\mathcal{E}_{\eta}^{\prime \prime} w=-\frac{i}{2} \partial_{x} w-2 T_{|\eta|^{2}} w-H_{\eta^{2}} w+\frac{1}{4} w
$$

In [7], Holmer and Zworski consider the case of the nonlinear cubic Schrödinger equation with a Dirac potential, that can be generalized to the case of a multiplicative linear potential. The maximal time for which the approximation holds is of order $\frac{1}{\sqrt{\varepsilon}}$. Thus, the result we obtain for the Szegö equation with a Toeplitz potential (the natural extension of the multiplicative potential) is close to [7]. However, working with the Lyapunov functional as it was done in [7] does not give the desired result in the case of the Szegö equation, since we no longer have a Galilean 
invariance. Consequently, we use the linearized operator, as it was done by the above cited authors in [8], in the case of a slowly varying potential.

Notice that the exact effective dynamics given by $\bar{a}, \bar{\alpha}, \bar{\phi}, \bar{\mu}$, are an approximation of the solution of the perturbed equation only for times

$$
0<t \leq \frac{\delta}{6 \ln c_{0}} \cdot \frac{1}{\varepsilon^{\frac{1}{2}-\delta}} \ln \left(\frac{1}{\varepsilon}\right) \leq \frac{\delta}{6 \ln c_{0}} \cdot \frac{1}{\varepsilon^{\frac{1}{5}}} \ln \left(\frac{1}{\varepsilon}\right),
$$

where $\delta>\frac{3}{10}$. (If we agree to have an approximation of order $\varepsilon^{\frac{1}{2}}$, instead of that of order $\varepsilon^{\frac{1}{2}+\frac{\delta}{3}}$ that we have, we can actually go up to times $0<t \leq \frac{\delta}{6 \ln c_{0}}$. $\frac{1}{\varepsilon^{\frac{1}{4}}} \ln \left(\frac{1}{\varepsilon}\right)$.) For larger times, the approximation is only given by $a, \alpha, \phi, \mu$, which are perturbations of the effective dynamics. The fact that we cannot approximate the solution by the exact effective dynamics for larger times (i.e. $0<\delta<\frac{3}{10}$ ) is due to the estimate on $|\phi-\bar{\phi}|$ which is only of order $O\left(\varepsilon^{2 \delta-}\right)$, while we need an approximation of order $O\left(\varepsilon^{\frac{1}{2}+\frac{\delta}{3}}\right)$. This difficulty is caused by the complicated form of the effective dynamics and by the fact that the perturbed equation does not conserve the momentum $\|u\|_{\dot{H}^{1 / 2}}^{2}$. In the case of the nonlinear Schrödinger equation with a Dirac or a slowly varying potential, the effective dynamics have a simpler form and give a good approximation of the solution for all the range of times considered in $[\mathbf{7}, \mathbf{8}]$.

The structure of the paper is as follows. In section 2 we briefly describe the manifold of solitons. In section 3 we find the effective dynamics. In section 4 we use the implicit function theorem to prove the orthogonal decomposition of the flow and determine the equation of $w$, the part of the flow which is orthogonal to $M$. In section 5 we prove the coerciveness of the linearized operator in directions orthogonal to the manifold $M$. In section 6 we estimate $w$ using a bootstrap argument and in section 7 we conclude the proof of Theorem 1.1.

\section{Manifold of solitons}

We introduce below the manifold of solitons for the Szegö equation on the real line.

For $g=(a, \alpha, \phi, \mu) \in \mathbb{R} \times \mathbb{R}_{+}^{*} \times \mathbb{T} \times \mathbb{R}_{+}^{*}$, where $\mathbb{T}=\mathbb{R} / 2 \pi \mathbb{Z}$, we define the following map on $L_{+}^{2}(\mathbb{R})$

$$
u \mapsto g \cdot u, \quad g \cdot u(x):=e^{i \phi} \alpha \mu u(\mu(x-a)) .
$$

This action gives a group structure on $\mathbb{R} \times \mathbb{R}_{+}^{*} \times \mathbb{T} \times \mathbb{R}_{+}^{*}$ :

$$
(a, \alpha, \phi, \mu) \cdot\left(a^{\prime}, \alpha^{\prime}, \phi^{\prime}, \mu^{\prime}\right)=\left(a^{\prime \prime}, \alpha^{\prime \prime}, \phi^{\prime \prime}, \mu^{\prime \prime}\right),
$$

where

$$
\left\{\begin{array}{l}
a^{\prime \prime}=a+\frac{a^{\prime}}{\mu} \\
\alpha^{\prime \prime}=\alpha \alpha^{\prime} \\
\phi^{\prime \prime}=\phi+\phi^{\prime} \\
\mu^{\prime \prime}=\mu \mu^{\prime} .
\end{array}\right.
$$


We denote this group by $G$. In order to determine the Lie algebra $\mathfrak{g}$ corresponding to this Lie group, we compute

$$
\begin{aligned}
& \left.\partial_{a}[(a, 1,0,1) \cdot u]\right|_{a=0}=-\partial_{x} u \\
& \left.\partial_{\alpha}[(0, \alpha, 0,1) \cdot u]\right|_{\alpha=1}=u \\
& \left.\partial_{\phi}[(0,1, \phi, 1) \cdot u]\right|_{\phi=0}=i u \\
& \left.\partial_{\mu}[(0,1,0, \mu) \cdot u]\right|_{\mu=1}=x \partial_{x} u+u=\partial_{x}(x \cdot u) .
\end{aligned}
$$

Then, the Lie algebra $\mathfrak{g}$ is generated by

$$
e_{1}=-\partial_{x}, \quad e_{2}=1, \quad e_{3}=i, e_{4}=\partial_{x} \cdot x .
$$

It acts on $\cup_{N \in \mathbb{N}} \mathcal{M}(N)$, where

$\mathcal{M}(N):=\left\{\frac{A(z)}{B(z)} \in L_{+}^{2} \mid \operatorname{deg}(B)=N, \operatorname{deg}(A) \leq N-1, B(0)=1, \operatorname{pgcd}(A, B)=1\right\}$.

Notice that according to [12][Lemma 6.2.1], we have that $\cup_{N \in \mathbb{N}} \mathcal{M}(N)$ is dense in $L_{+}^{2}(\mathbb{R})$.

The action $g$ is conformally symplectic in the sense that

$$
g^{*} \omega=\alpha^{2}(g) \mu(g) \omega .
$$

Indeed, with the change of variables $y=\mu(x-a)$

$$
\begin{aligned}
\left(g^{*} \omega\right)(u, v) & =\operatorname{Im} \int_{\mathbb{R}} e^{i \phi} \alpha \mu u(\mu(x-a)) e^{-i \phi} \alpha \mu \bar{v}(\mu(x-a)) d x \\
& =\alpha^{2} \mu \operatorname{Im} \int_{\mathbb{R}} u(y) \bar{v}(y) d y=\alpha^{2} \mu \omega(u, v) .
\end{aligned}
$$

DeFINITION 2. The manifold of solitons is the orbit of $\eta, \eta(x)=\frac{1}{x+i}$, under the action of the group $G$ :

$$
M=G \cdot \eta=\left\{e^{i \phi} \alpha \mu \eta(\mu(x-a)), \phi, a \in \mathbb{R}, \alpha>0, \mu>0\right\} .
$$

We then make the following identifications:

$$
M=G \cdot \eta \simeq G, T_{\eta} M=\mathfrak{g} \cdot \eta \simeq \mathfrak{g} .
$$

For $b=0$, the flow of $H_{0}$ is tangent to the manifold of solitons $M$. This corresponds to the fact that if $u(0, x) \in M$, then $u(t, x) \in M$ for all $t \in \mathbb{R}$. More precisely, by equations (1.5) and (1.6), we have that if $u(0, x)=e^{i \phi} \alpha \mu \eta(\mu(x-a))$, then

$$
u(t, x)=g(t) \cdot \eta=e^{i \phi(t)} \alpha(t) \mu(t) \eta(\mu(t)(x-a(t)),
$$

where

$$
\left\{\begin{array}{l}
\dot{a}(t)=\frac{\alpha^{2} \mu}{2} \\
\dot{\alpha}(t)=0 \\
\dot{\phi}(t)=-\frac{\alpha^{2} \mu^{2}}{4} \\
\dot{\mu}(t)=0 .
\end{array}\right.
$$




\section{Effective dynamics}

We will compute in this section the restriction to the manifold of solitons $M$ of the symplectic form $\left.\omega\right|_{M}$ and prove that $\left(M,\left.\omega\right|_{M}\right)$ is a symplectic manifold. Then, we compute the restriction of the Hamiltonian $\left.H_{b}\right|_{M}$, as well as the vector field associated to $\left.H_{b}\right|_{M}$. This vector field yields a flow on the manifold of solitons $M$, that we refer to as the effective dynamics.

First we compute $\left(\left.\omega\right|_{M}\right)_{\eta}$ on $T_{\eta} M$, at the point $\eta$. Using

$$
\left(\left.\omega\right|_{M}\right)_{\eta}\left(e_{i}, e_{j}\right)=\operatorname{Im} \int_{\mathbb{R}}\left(e_{i} \cdot \eta\right)(x) \overline{\left(e_{j} \cdot \eta\right)(x)} d x,
$$

and the residue theorem, we get

$$
\begin{aligned}
& \left(\left.\omega\right|_{M}\right)_{\eta}\left(e_{1}, e_{2}\right)=-\operatorname{Im} \int_{\mathbb{R}} \partial_{x}\left(\frac{1}{x+i}\right) \overline{\frac{1}{x+i}} d x=-\frac{\pi}{2} \\
& \left(\left.\omega\right|_{M}\right)_{\eta}\left(e_{1}, e_{3}\right)=0, \quad\left(\left.\omega\right|_{M}\right)_{\eta}\left(e_{1}, e_{4}\right)=-\frac{\pi}{2}, \quad\left(\left.\omega\right|_{M}\right)_{\eta}\left(e_{2}, e_{3}\right)=-\pi \\
& \left(\left.\omega\right|_{M}\right)_{\eta}\left(e_{2}, e_{4}\right)=0, \quad\left(\left.\omega\right|_{M}\right)_{\eta}\left(e_{3}, e_{4}\right)=\frac{\pi}{2} .
\end{aligned}
$$

Hence

$$
\left(\left.\omega\right|_{M}\right)_{\eta}=\frac{\pi}{2}(d \alpha \wedge d a+d \mu \wedge d a+2 d \phi \wedge d \alpha+d \phi \wedge d \mu) .
$$

Let us now compute $\left(\left.\omega\right|_{M}\right)_{g \cdot \eta}$ for arbitrary $g \in G$. By (2.3) we can identify the action of $g$ on $M$ with the action $g: G \rightarrow G$ given by (2.1). Then, we have that the differential $d_{\eta} g: T_{\eta} M \rightarrow T_{g \cdot \eta} M$ is given by

$$
d_{\eta} g=\frac{1}{\mu} d a+\alpha d \alpha+d \phi+\mu d \mu .
$$

By equation (2.2), we have that

$$
\omega_{g \cdot \eta}\left(d_{\eta} g(u), d_{\eta} g(v)\right)=\alpha^{2} \mu \omega_{\eta}(u, v) .
$$

Then, equations (3.2), (3.3), and (3.1) yield

$$
\begin{array}{r}
\left(\left.\omega\right|_{M}\right)_{g \cdot \eta}\left(X_{1}\left(\frac{\partial}{\partial a}\right)_{g \cdot \eta}+X_{2}\left(\frac{\partial}{\partial \alpha}\right)_{g \cdot \eta}+X_{3}\left(\frac{\partial}{\partial \phi}\right)_{g \cdot \eta}+X_{4}\left(\frac{\partial}{\partial \mu}\right)_{g \cdot \eta}\right. \\
\left.Y_{1}\left(\frac{\partial}{\partial a}\right)_{g \cdot \eta}+Y_{2}\left(\frac{\partial}{\partial \alpha}\right)_{g \cdot \eta}+Y_{3}\left(\frac{\partial}{\partial \phi}\right)_{g \cdot \eta}+Y_{4}\left(\frac{\partial}{\partial \mu}\right)_{g \cdot \eta}\right) \\
=\alpha^{2} \mu\left(\left.\omega\right|_{M}\right)_{\eta}\left(\mu X_{1}\left(\frac{\partial}{\partial a}\right)_{\eta}+\frac{X_{2}}{\alpha}\left(\frac{\partial}{\partial \alpha}\right)_{\eta}+X_{3}\left(\frac{\partial}{\partial \phi}\right)_{\eta}+\frac{X_{4}}{\mu}\left(\frac{\partial}{\partial \mu}\right)_{\eta},\right. \\
\left.\mu Y_{1}\left(\frac{\partial}{\partial a}\right)_{\eta}+\frac{Y_{2}}{\alpha}\left(\frac{\partial}{\partial \alpha}\right)_{\eta}+Y_{3}\left(\frac{\partial}{\partial \phi}\right)_{\eta}+\frac{Y_{4}}{\mu}\left(\frac{\partial}{\partial \mu}\right)_{\eta}\right) \\
=\frac{\pi}{2} \alpha^{2} \mu\left(\frac{\mu}{\alpha} d \alpha \wedge d a+d \mu \wedge d a+\frac{2}{\alpha} d \phi \wedge d \alpha+\frac{1}{\mu} d \phi \wedge d \mu\right) \\
\left(X_{1}\left(\frac{\partial}{\partial a}\right)_{\eta}+X_{2}\left(\frac{\partial}{\partial \alpha}\right)_{\eta}+X_{3}\left(\frac{\partial}{\partial \phi}\right)_{\eta}+X_{4}\left(\frac{\partial}{\partial \mu}\right)_{\eta}\right. \\
\left.Y_{1}\left(\frac{\partial}{\partial a}\right)_{\eta}+Y_{2}\left(\frac{\partial}{\partial \alpha}\right)_{\eta}+Y_{3}\left(\frac{\partial}{\partial \phi}\right)_{\eta}+Y_{4}\left(\frac{\partial}{\partial \mu}\right)_{\eta}\right) .
\end{array}
$$


Thus,

$$
\left.\omega\right|_{M}=\alpha^{2} \mu \frac{\pi}{2}\left(\frac{\mu}{\alpha} d \alpha \wedge d a+d \mu \wedge d a+\frac{2}{\alpha} d \phi \wedge d \alpha+\frac{1}{\mu} d \phi \wedge d \mu\right) .
$$

One can easily verify that $\left.\omega\right|_{M}$ is a non-degenerate symplectic form and therefore, $\left(M,\left.\omega\right|_{M}\right)$ is a symplectic manifold.

Let $f$ be a function defined on $M \simeq G$. Then, $f$ admits a Hamiltonian vector field $X_{f}$ on $M$ if

$$
\left.\omega\right|_{M}\left(\cdot, X_{f}\right)=d f=f_{a} d a+f_{\alpha} d \alpha+f_{\mu} d \mu+f_{\phi} d \phi,
$$

where $f_{a}=\frac{\partial f}{\partial_{a}}$ and $f_{\alpha}, f_{\phi}$, and $f_{\mu}$ are defined similarly. Denoting $X_{f}=X_{1} \frac{\partial}{\partial a}+$ $X_{2} \frac{\partial}{\partial \alpha}+X_{3} \frac{\partial}{\partial \phi}+X_{4} \frac{\partial}{\partial \mu}$ and using (3.4), the above equation is equivalent to

$$
\begin{aligned}
& \alpha^{2} \mu \frac{\pi}{2}\left(\frac{\mu}{\alpha}\left(X_{1} d \alpha-X_{2} d a\right)+\left(X_{1} d \mu-X_{4} d a\right)\right. \\
&+\frac{2}{\alpha}\left(X_{2} d \phi-X_{3} d \alpha\right)+\left.\frac{1}{\mu}\left(X_{4} d \phi-X_{3} d \mu\right)\right) \\
&=f_{a} d a+f_{\alpha} d \alpha+f_{\mu} d \mu+f_{\phi} d \phi
\end{aligned}
$$

Then, the components of the vector field $X_{f}$ are

$$
\left\{\begin{array}{l}
X_{1}=-\frac{2}{\alpha^{2} \mu^{2} \pi}\left(-2 \mu f_{\mu}+\alpha f_{\alpha}\right) \\
X_{2}=\frac{2}{\alpha^{2} \mu^{2} \pi}\left(\alpha f_{a}+\alpha \mu f_{\phi}\right) \\
X_{3}=\frac{2}{\alpha^{2} \mu \pi}\left(\mu f_{\mu}-\alpha f_{\alpha}\right) \\
X_{4}=-\frac{2}{\alpha^{2} \mu \pi}\left(\mu f_{\phi}+2 f_{a}\right)
\end{array}\right.
$$

This allows us to determine the Hamiltonian flow associated to $X_{f}, \dot{u}=X_{f}(u)$, which is given by $(\dot{a}, \dot{\alpha}, \dot{\phi}, \dot{\mu})=\left(X_{1}, X_{2}, X_{3}, X_{4}\right)$.

Let us now compute $\left.H_{b}\right|_{M}$ and find its Hamiltonian vector field.

$$
\begin{aligned}
\left.H_{b}\right|_{M}(g \cdot \eta) & =\frac{1}{4} \int_{\mathbb{R}} \alpha^{4} \mu^{4}|\eta(\mu(x-a))|^{4} d x+\frac{\varepsilon}{2} \int_{\mathbb{R}} b(x) \alpha^{2} \mu^{2}|\eta(\mu(x-a))|^{2} d x \\
& =\frac{\alpha^{4} \mu^{3}}{4} \int_{\mathbb{R}}|\eta(x)|^{4} d x+\frac{\varepsilon \alpha^{2} \mu^{2}}{2} \int_{\mathbb{R}} b(x)|\eta(\mu(x-a))|^{2} d x \\
& =\frac{\alpha^{4} \mu^{3} \pi}{8}+\frac{\varepsilon \alpha^{2} \mu}{2} \int_{\mathbb{R}} b\left(a+\frac{x}{\mu}\right)|\eta(x)|^{2} d x .
\end{aligned}
$$

Taking $f=\left.H_{b}\right|_{M}$, we have that

$$
\left\{\begin{array}{l}
f_{a}=\frac{\varepsilon \alpha^{2} \mu}{2} \int b^{\prime}\left(a+\frac{x}{\mu}\right)|\eta(x)|^{2} d x \\
f_{\alpha}=\frac{\pi \alpha^{3} \mu^{3}}{2}+\varepsilon \alpha \mu \int b\left(a+\frac{x}{\mu}\right)|\eta(x)|^{2} d x \\
f_{\phi}=0 \\
f_{\mu}=\frac{3 \pi \alpha^{4} \mu^{2}}{8}+\frac{\varepsilon \alpha^{2}}{2} \int b\left(a+\frac{x}{\mu}\right)|\eta(x)|^{2} d x-\frac{\varepsilon \alpha^{2}}{2} \int b^{\prime}\left(a+\frac{x}{\mu}\right) \frac{x}{\mu}|\eta(x)|^{2} d x .
\end{array}\right.
$$


As above, we determine the components of the Hamiltonian vector field associated to $f=\left.H_{b}\right|_{M}$, and obtain that the flow of $\left.H_{b}\right|_{M}$ is given by

$$
\left\{\begin{array}{l}
\dot{a}=\frac{\alpha^{2} \mu}{2}-\frac{2 \varepsilon}{\pi \mu} \int b^{\prime}\left(a+\frac{x}{\mu}\right) \frac{x}{\mu}|\eta(x)|^{2} d x \\
\dot{\alpha}=\frac{\varepsilon \alpha}{\pi \mu} \int b^{\prime}\left(a+\frac{x}{\mu}\right)|\eta(x)|^{2} d x \\
\dot{\phi}=-\frac{\alpha^{2} \mu^{2}}{4}-\frac{\varepsilon}{\pi} \int b\left(a+\frac{x}{\mu}\right)|\eta(x)|^{2} d x-\frac{\varepsilon}{\pi} \int b^{\prime}\left(a+\frac{x}{\mu}\right) \frac{x}{\mu}|\eta(x)|^{2} d x \\
\dot{\mu}=-\frac{2 \varepsilon}{\pi} \int b^{\prime}\left(a+\frac{x}{\mu}\right)|\eta(x)|^{2} d x .
\end{array}\right.
$$

\section{Reparametrized evolution}

Our goal is to show that the flow generated by $H_{b}$ can be approximated by the effective flow of $\left.H_{b}\right|_{M}$. In order to do so, we decompose the solution $u(t)$ of the Szegö equation with small Toeplitz potential (1.4), into a component belonging to $M$ and a component which is symplectically orthogonal to $M$ in the sense that:

$$
u(t)=g(t) \cdot(\eta+w(t)), \quad \omega(w(t), X \eta)=0, \forall X \in \mathfrak{g} .
$$

The key point is to prove that the orthogonal component $w$ is small.

Let us show that the above decomposition/reparametrization is indeed possible at least for short time.

LEMMA 4.1. For a compact subset $\Sigma$ of $\mathbb{R} \times \mathbb{R}_{+}^{*} \times \mathbb{T} \times \mathbb{R}_{+}^{*}$ and $\gamma>0$, denote by

$$
U_{\Sigma, \gamma}=\left\{u \in H^{\frac{1}{2}}+; \inf _{g \in \Sigma}\|u-g \cdot \eta\|_{H_{+}^{\frac{1}{2}}}<\gamma\right\} .
$$

a $\gamma$-tubular neighborhood of $\Sigma$.

There exists $\gamma_{0}=\gamma_{0}(\Sigma)$ such that if $u \in U_{\Sigma, \gamma}$, with $\gamma \leq \gamma_{0}$, then there exists a unique element $g(u) \in \Sigma$ with the property

$$
\omega\left(g(u)^{-1} \cdot u-\eta, X \cdot \eta\right)=0, \forall X \in \mathfrak{g} .
$$

Proof. Consider the function $F: H_{+}^{\frac{1}{2}} \times G \rightarrow \mathfrak{g}^{*}$,

$$
F(u, h)(X)=\omega(h \cdot u-\eta, X \cdot \eta) .
$$

We want to solve $F(u, h)=0$ for $h=h(u)$. We verify that the function $F$ satisfies the hypotheses of the Implicit Function Theorem:

(i) $F(u, h)$ is of class $C^{1}$ in $h$,

(ii) $F\left(g \cdot \eta, g^{-1}\right)=0$ for all $g \in G$,

(iii) $d_{h} F\left(g \cdot \eta, g^{-1}\right): T_{g^{-1}} G \rightarrow \mathfrak{g}^{*}$ is invertible for all $g \in G$.

The first two properties can be checked directly. As for the third property, it is enough to check it for $g=e=(1,0,1,0)$, the unity of the group $G$. Thus, since $T_{e} G=\mathfrak{g}$, it is enough to check that $d_{h} F(\eta, e): \mathfrak{g} \rightarrow \mathfrak{g}^{*}$ is invertible. But

$d_{h} F(\eta, e)=\left(\left.\omega\right|_{M}\right)_{\eta}$ which is non-degenerate because, in the basis $\left\{e_{j} \cdot \eta\right\}_{j=1}^{4}$ of $\mathfrak{g}$, it writes

$$
\frac{\pi}{2}\left(\begin{array}{cccc}
0 & -1 & 0 & -1 \\
1 & 0 & -2 & 0 \\
0 & 2 & 0 & 1 \\
1 & 0 & -1 & 0
\end{array}\right)
$$

whose determinant does not vanish.

Thus, the orthogonal decomposition (4.1), with $w(t)=g(t)^{-1} \cdot u(t)-\eta$, holds as long as $u(t)$ is close enough to $M=G \cdot \eta$.

In order to find the equation that $w$ satisfies, we need the following lemmas: 
LEMMA 4.2. If $t \mapsto g(t)=(a(t), \alpha(t), \phi(t), \mu(t))$ is a $C^{1}$ function and $u \in \cup_{N \in \mathbb{N}} \mathcal{M}(N)$, then

$$
\frac{d}{d t} g(t) \cdot u=g(t) \cdot(Y(t) u)
$$

where $Y(t)=\dot{a}(t) \mu(t) e_{1}+\frac{\dot{\alpha}(t)}{\alpha(t)} e_{2}+\dot{\phi}(t) e_{3}+\frac{\dot{\mu}(t)}{\mu(t)} e_{4}$.

PROOF.

$$
\begin{aligned}
\frac{d}{d t} g(t) \cdot u= & \frac{d}{d t}\left(e^{i \phi} \alpha \mu u(\mu(x-a))\right) \\
= & \dot{\phi} e^{i \phi} \alpha \mu u(\mu(x-a))+e^{i \phi} \dot{\alpha} \mu u(\mu(x-a))+e^{i \phi} \alpha \dot{\mu} u(\mu(x-a)) \\
& +e^{i \phi} \alpha \mu \partial_{x} u(\mu(x-a)) \dot{\mu} x-e^{i \phi} \alpha \mu \partial_{x} u(\mu(x-a))(\dot{\mu} a+\mu \dot{a}) \\
= & \dot{\phi} g \cdot\left(e_{3} \cdot u\right)+\frac{\dot{\alpha}}{\alpha} g \cdot\left(e_{2} \cdot u\right)+\frac{\dot{\mu}}{\mu} g \cdot\left(e_{4} \cdot u\right)+\dot{a} \mu g \cdot\left(e_{1} \cdot u\right) \\
= & g \cdot(Y(t) u) .
\end{aligned}
$$

We also need Lemma 2.1 from [8], that we restate in the context of our problem.

Lemma 4.3. Suppose that $g: H_{+}^{\frac{1}{2}} \rightarrow H_{+}^{\frac{1}{2}}$ is a diffeomorphism such that $g^{*} \omega=\rho(g) \omega$, where $\rho(g) \in C^{\infty}\left(H_{+}^{\frac{1}{2}}, \mathbb{R}^{*}\right)$. Then, for $f \in C^{\infty}\left(H_{+}^{\frac{1}{2}}, \mathbb{R}\right)$ we have that

$$
\left(g^{-1}\right)_{*} X_{f}(g(\rho))=\frac{1}{\rho(g)} X_{g^{*} f}(\rho), \rho \in H_{+}^{\frac{1}{2}}
$$

In the next proposition we determine the equation satisfied by $w$.

Proposition 4.4. If the solution of the perturbed Szegö equation (1.7) can be reparametrized as in Lemma 4.1, $u(t)=g(t) \cdot(\eta+w(t))$, for all $t$ in an interval $\left(t_{1}, t_{2}\right)$, then $w$ satisfies the following equation:

$$
\begin{aligned}
\partial_{t} w & =-X \eta+\left(-i \varepsilon \Pi\left(b\left(a+\frac{x}{\mu}\right) \eta\right)+2 B e_{1} \cdot \eta-C e_{2} \cdot \eta+(A+B) e_{3} \cdot \eta+2 C e_{4} \cdot \eta\right) \\
& -X w+\left(-i \varepsilon \Pi\left(b\left(a+\frac{x}{\mu}\right) w\right)+2 B e_{1} \cdot w-C e_{2} \cdot w+(A+B) e_{3} \cdot w+2 C e_{4} \cdot w\right) \\
& +i \alpha^{2} \mu^{2} \mathcal{L} w-i \alpha^{2} \mu^{2} \mathcal{N} w
\end{aligned}
$$


where

$$
\begin{aligned}
& X:=\left(\dot{a} \mu-\frac{\alpha^{2} \mu^{2}}{2}+2 B\right) e_{1}+\left(\frac{\dot{\alpha}}{\alpha}-C\right) e_{2}+\left(\dot{\phi}+\frac{\alpha^{2} \mu^{2}}{4}+A+B\right) e_{3} \\
&+\left(\frac{\dot{\mu}}{\mu}+2 C\right) e_{4}, \\
& \mathcal{L} w:=-\frac{i}{2} \partial_{x} w-2 T_{|\eta|^{2}} w-H_{\eta^{2}} w+\frac{1}{4} w, \\
& \mathcal{N} w:=\Pi\left(|w|^{2} w+|w|^{2} \eta+2 w \operatorname{Re}(\eta \bar{w})\right), \\
& A:=\frac{\varepsilon}{\pi} \int b\left(a+\frac{x}{\mu}\right)|\eta(x)|^{2} d x \\
& B:=\frac{\varepsilon}{\pi} \int b^{\prime}\left(a+\frac{x}{\mu}\right) x|\eta(x)|^{2} \frac{d x}{\mu} \\
& C:=\frac{\varepsilon}{\pi} \int b^{\prime}\left(a+\frac{x}{\mu}\right)|\eta(x)|^{2} \frac{d x}{\mu} .
\end{aligned}
$$

Proof. Denote $\tilde{u}=w+\eta=g^{-1} u$. Then, by Lemma 4.2, we have that

$$
\partial_{t} u=\partial_{t}(g \cdot(\eta+w))=g \cdot Y(\eta+w)+g \cdot \partial_{t} w .
$$

Then, Lemma 4.3 yields

$$
\begin{aligned}
\partial_{t} w & =-Y(\eta+w)+g^{-1} \partial_{t} u \\
& =-Y(\eta+w)+g^{-1} X_{H_{b}}(u)=-Y(\eta+w)+g^{-1} X_{H_{b}}(g \tilde{u}) \\
& =-Y(\eta+w)+\frac{1}{\alpha^{2} g} X_{g^{*} H_{b}}(\tilde{u}) .
\end{aligned}
$$

Since

$$
\left(g^{*} H_{b}\right)(\tilde{u})=H_{b}(g \tilde{u})=\frac{\alpha^{4} \mu^{3}}{4} \int|\tilde{u}|^{4} d x+\frac{\varepsilon \alpha^{2} \mu}{2} \int b\left(a+\frac{x}{\mu}\right)|\tilde{u}|^{2} d x,
$$

we have that

$$
\left(X_{g^{*} H_{b}}\right)(\tilde{u})=-i \Pi\left(\alpha^{4} \mu^{3}|\tilde{u}|^{2} \tilde{u}+\varepsilon \alpha^{2} \mu b\left(a+\frac{x}{\mu}\right) \tilde{u}\right)
$$

and therefore,

$$
\begin{aligned}
(4.3) \partial_{t} w= & -Y(\eta+w)-\frac{i}{\alpha^{2} \mu} \Pi\left(\alpha^{4} \mu^{3}|\eta+w|^{2}(\eta+w)+\varepsilon \alpha^{2} \mu b\left(a+\frac{x}{\mu}\right)(\eta+w)\right) \\
= & \left(-Y \eta-i \varepsilon \Pi\left(b\left(a+\frac{x}{\mu}\right) \eta\right)\right)+\left(-Y w-i \varepsilon \Pi\left(b\left(a+\frac{x}{\mu}\right) w\right)\right) \\
& -i \alpha^{2} \mu^{2} \Pi\left(2 \operatorname{Re}(\eta \bar{w}) \eta+|\eta|^{2} w\right) \\
& -i \alpha^{2} \mu^{2} \Pi\left(|w|^{2} w+2 \operatorname{Re}(\eta \bar{w}) w+|w|^{2} \eta\right)-i \alpha^{2} \mu^{2} \Pi\left(|\eta|^{2} \eta\right) .
\end{aligned}
$$

Denoting

$$
X=Y+\left(-\frac{\alpha^{2} \mu^{2}}{2}+2 B\right) e_{1}-C e_{2}+\left(\frac{\alpha^{2} \mu^{2}}{4}+A+B\right) e_{3}+2 C e_{4},
$$

and noticing that

$$
-\frac{\alpha^{2} \mu^{2}}{2} e_{1} \cdot \eta=\frac{\alpha^{2} \mu^{2}}{2} \partial_{x} \eta, \quad \frac{\alpha^{4} \mu^{3}}{4} e_{3} \cdot \eta=\frac{i}{4} \alpha^{4} \mu^{3} \eta,
$$


and similar relations hold for $w$, we obtain

$$
\begin{aligned}
\partial_{t} w= & -X \eta+\left(-i \varepsilon \Pi\left(b\left(a+\frac{x}{\mu}\right) \eta\right)+2 B e_{1} \cdot \eta-C e_{2} \cdot \eta\right. \\
& \left.+(A+B) e_{3} \cdot \eta+2 C e_{4} \cdot \eta\right) \\
& -X w+\left(-i \varepsilon \Pi\left(b\left(a+\frac{x}{\mu}\right) w\right)+2 B e_{1} \cdot w-C e_{2} \cdot w\right. \\
& \left.+(A+B) e_{3} \cdot w+2 C e_{4} \cdot w\right) \\
& -i \alpha^{2} \mu^{2}\left(\Pi\left(2|\eta|^{2} w+\eta^{2} \bar{w}\right)+\frac{i}{2} \partial_{x} w-\frac{w}{4}\right)-i \alpha^{2} \mu^{2}\left(\Pi\left(|\eta|^{2} \eta\right)+\frac{i}{2} \partial_{x} \eta-\frac{\eta}{4}\right) \\
& -i \alpha^{2} \mu^{2} \Pi\left(|w|^{2} w+2 \operatorname{Re}(\eta \bar{w}) w+|w|^{2} \eta\right) .
\end{aligned}
$$

Equation (1.13) and (1.14) yield the conclusion.

REMARK 4.5. Notice that $X \equiv 0$ is equivalent to $a, \alpha, \phi, \mu$ satisfying the effective dynamics (1.9).

LEMMA 4.6. If the solution of the perturbed Szegö equation (1.7) can be reparametrized as in Lemma 4.1, $u(t)=g(t) \cdot(\eta+w(t))$ at time $t$, then the $L^{2}$-norm of $w(t)$ is equal to

$$
\|w(t)\|_{L^{2}}^{2}=\pi\left(\frac{\alpha_{0}^{2} \mu_{0}}{\alpha^{2}(t) \mu(t)}-1\right) .
$$

Consequently, $\alpha^{2}(t) \mu(t) \leq \alpha_{0}^{2} \mu_{0}$.

Proof. By the conservation of the $L^{2}$-norm of the solution of the Szegö equation with a Toeplitz potential, we have that

$$
\|\eta+w(t)\|_{L^{2}}^{2}=\left\|g(t)^{-1} u(t)\right\|_{L^{2}}^{2}=\frac{1}{\alpha^{2}(t) \mu(t)}\|u(t)\|_{L^{2}}^{2}=\frac{\|u(0)\|_{L^{2}}^{2}}{\alpha^{2}(t) \mu(t)}=\frac{\pi \alpha_{0}^{2} \mu_{0}}{\alpha^{2}(t) \mu(t)} .
$$

By the orthogonality of $w$ and $\eta$, we have that $\omega(w, X \cdot \eta)=0$, for all $X \in \mathfrak{g}$. In particular, taking $X=e_{3}$, we obtain

$$
\langle w, \eta\rangle=\operatorname{Re} \int w \bar{\eta} d x=-\operatorname{Im} \int w \overline{i \eta} d x=-\omega\left(w, e_{3} \cdot \eta\right)=0 .
$$

Then

$$
\|\eta+w(t)\|_{L^{2}}^{2}=\|\eta\|_{L^{2}}^{2}+\|w(t)\|_{L^{2}}^{2}=\pi+\|w(t)\|_{L^{2}}^{2}
$$

and the conclusion follows.

Next we define $P$, the symplectically orthogonal projection on the manifold of solitons $M$. We also give two technical lemmas concerning some properties of $P$.

Definition 3. Define the projection onto $T_{\eta} M=\mathfrak{g} \cdot \eta \simeq \mathfrak{g}$ by $P:\left(\cup_{N \in \mathbb{N}} \mathcal{M}(N)\right)^{\prime} \rightarrow \mathfrak{g}$,

$$
\omega(u-P(u) \eta, Y \eta)=0, \forall Y \in \mathfrak{g} .
$$


LEMMA 4.7. Let $\|\cdot\|$ be a norm on $\mathfrak{g}$ obtained by using the standard $\mathbb{R}^{4}$ norm in the basis $\left\{e_{1}, e_{2}, e_{3}, e_{4}\right\}$. Then, for all $w \in H_{+}^{\frac{1}{2}}$ and $Y \in \mathfrak{g}$, we have

$$
\begin{aligned}
& \|P(Y w)\| \leq C\|Y\|\|w\|_{L^{2}} \\
& \|P(i \mathcal{N} w)\| \leq C\|w\|_{H_{+}^{\frac{1}{2}}}^{2}\left(\|w\|_{H_{+}^{\frac{1}{2}}}+1\right)
\end{aligned}
$$

Proof. Let $P=\sum_{j=1}^{4} P_{j} e_{j}, P_{j}: H_{+}^{-\frac{1}{2}} \rightarrow \mathbb{R}$. Then the definition of $P$ yields

$$
\left(\left.\omega\right|_{M}\right)_{\eta}\left(u-\sum_{j=1}^{4} P_{j} e_{j} \cdot \eta, a_{1} e_{1} \cdot \eta+a_{2} e_{2} \cdot \eta+a_{3} e_{3} \cdot \eta+a_{4} e_{4} \cdot \eta\right)=0
$$

for all $a_{i} \in \mathbb{R}$. Then, it follows that

$$
\begin{aligned}
& a_{1}\left(\omega\left(u, e_{1} \cdot \eta\right)-\frac{\pi}{2} P_{2}-\frac{\pi}{2} P_{4}\right)+a_{2}\left(\omega\left(u, e_{2} \cdot \eta\right)+\frac{\pi}{2} P_{1}-\pi P_{3}\right) \\
& +a_{3}\left(\omega\left(u, e_{3} \cdot \eta\right)+\pi P_{2}+\frac{\pi}{2} P_{4}\right)+a_{4}\left(\omega\left(u, e_{4} \cdot \eta\right)+\frac{\pi}{2} P_{1}-\frac{\pi}{2} P_{3}\right)=0
\end{aligned}
$$

for all $a_{i} \in \mathbb{R}$. Therefore,

$$
\left\{\begin{array}{l}
P_{1}(u)=\frac{2}{\pi}\left(\omega\left(u, e_{2} \cdot \eta\right)-2 \omega\left(u, e_{4} \cdot \eta\right)\right) \\
P_{2}(u)=\frac{2}{\pi}\left(-\omega\left(u, e_{3} \cdot \eta\right)-\omega\left(u, e_{1} \cdot \eta\right)\right) \\
P_{3}(u)=\frac{2}{\pi}\left(\omega\left(u, e_{2} \cdot \eta\right)-\omega\left(u, e_{4} \cdot \eta\right)\right) \\
P_{4}(u)=\frac{2}{\pi}\left(2 \omega\left(u, e_{1} \cdot \eta\right)+\omega\left(u, e_{3} \cdot \eta\right)\right)
\end{array}\right.
$$

The conclusion follows by using the Cauchy-Schwarz inequality and integration by parts. For example, for $P_{1}$ we have

$$
\begin{aligned}
\left\|P_{1}(Y w)\right\| \leq & \left|\int Y w \bar{\eta}\right|+2\left|\int Y w \overline{\partial_{x}(x \eta)}\right| \\
= & \left|\int\left(-Y_{1} \partial_{x} w+Y_{2} w+i Y_{3} w+Y_{4} \partial_{x}(x w)\right) \bar{\eta} d x\right| \\
& +2\left|\int\left(-Y_{1} \partial_{x} w+Y_{2} w+i Y_{3} w+Y_{4} \partial_{x}(x w)\right) \overline{\partial_{x}(x \eta)} d x\right| \\
\leq & \|Y\|\left(\left|\int w \partial_{x} \bar{\eta} d x\right|+2\left|\int w \bar{\eta} d x\right|+\left|\int x w \partial_{x} \bar{\eta} d x\right|+2\left|\int w \partial_{x}^{2}(x \bar{\eta}) d x\right|\right. \\
& \left.+4\left|\int w \partial_{x}(x \bar{\eta}) d x\right|+2\left|\int x w \partial_{x}^{2}(x \bar{\eta}) d x\right|\right) \\
\leq & C\|Y\|\|w\|_{L^{2}}\left(\left\|\partial_{x} \eta\right\|_{L^{2}}\right. \\
& \left.+\|\eta\|_{L^{2}}+\left\|x \partial_{x} \eta\right\|_{L^{2}}+\left\|\partial_{x}^{2}(x \eta)\right\|_{L^{2}}+\left\|x \partial_{x}^{2}(x \eta)\right\|_{L^{2}}\right) \\
\leq & C\|Y\|\|w\|_{L^{2}} .
\end{aligned}
$$


By using the Sobolev embedding $H^{\frac{1}{2}}(\mathbb{R}) \subset L^{p}(\mathbb{R})$ for all $2 \leq p<\infty$, we have

$$
\begin{aligned}
\left\|P_{1}(i \mathcal{N} w)\right\|= & \left\|\omega(i \mathcal{N} w, \eta)-2 \omega\left(i \mathcal{N} w, \partial_{x}(x \eta)\right)\right\| \\
\leq & \left.\left|\int\right| w\right|^{2} w \bar{\eta} d x+\int|w|^{2}|\eta|^{2} d x+2 \int w \operatorname{Re}(\eta \bar{w}) \bar{\eta} d x \mid \\
& +\left.2\left|\int\right| w\right|^{2} w \partial_{x}(x \bar{\eta}) d x+\int|w|^{2} \eta \partial_{x}(x \bar{\eta}) d x \\
& +2 \int w \operatorname{Re}(\eta \bar{w}) \partial_{x}(x \bar{\eta}) d x \mid \\
\leq & C\left(\left\|w^{2}\right\|_{L^{2}}+\left\|w^{3}\right\|_{L^{2}}\right) \leq C\|w\|_{L^{4}}\left(\|w\|_{L^{4}}+\|w\|_{L^{8}}^{2}\right) \\
& \leq\|w\|_{H_{+}^{\frac{1}{2}}}^{2}\left(\|w\|_{H_{+}^{\frac{1}{2}}}+1\right) .
\end{aligned}
$$

LEMMA 4.8. If $f: \mathbb{R} \rightarrow \mathbb{R}$ is a function of class $C^{1}$ such that $f^{\prime} \in L^{1}(\mathbb{R}) \cap L^{2}(\mathbb{R})$ and $f \in L^{\infty}(\mathbb{R})$, then

$$
\begin{aligned}
P(\Pi(i f \eta)) & =\frac{2}{\pi}\left(\int f^{\prime}(x) x|\eta(x)|^{2} d x\right) e_{1}-\frac{1}{\pi}\left(\int f^{\prime}(x)|\eta(x)|^{2} d x\right) e_{2} \\
& +\frac{1}{\pi}\left(\int f(x)|\eta(x)|^{2} d x+\int f^{\prime}(x) x|\eta(x)|^{2} d x\right) e_{3} \\
& +\frac{2}{\pi}\left(\int f^{\prime}(x)|\eta(x)|^{2} d x\right) e_{4} .
\end{aligned}
$$

Proof. Let $Y=\sum_{j=1}^{4} a_{j} e_{j}$ be an arbitrary vector in $\mathfrak{g}$. Then, integrating by parts we have

$$
\begin{aligned}
\omega(\Pi(\text { if } \eta), Y \cdot \eta)=\omega\left(i f \eta, a_{1} e_{1} \cdot \eta+a_{2} e_{2} \cdot \eta+a_{3} e_{3} \cdot \eta+a_{4} e_{4} \cdot \eta\right) \\
=\operatorname{Im}\left(-a_{1} \int i f \eta \partial_{x} \bar{\eta} d x+a_{2} \int i f \eta \bar{\eta} d x\right. \\
\left.\quad+a_{3} \int i f \eta(-i) \bar{\eta} d x+a_{4} \int i f \eta \partial_{x}(x \bar{\eta}) d x\right) \\
=-\frac{a_{1}}{2} \int f \partial_{x}\left(|\eta|^{2}\right) d x+a_{2} \int f|\eta|^{2} d x \\
+a_{4} \operatorname{Re} \int f(x) \eta(x)\left(\bar{\eta}(x)+x \partial_{x} \bar{\eta}(x)\right) d x \\
=\frac{a_{1}}{2} \int f^{\prime}|\eta|^{2} d x+\left(a_{2}+a_{4}\right) \int f|\eta|^{2} d x-\frac{a_{4}}{2} \int\left(x f^{\prime}(x)+f(x)\right)|\eta(x)|^{2} d x \\
=\frac{a_{1}}{2} \int f^{\prime}|\eta|^{2} d x+\left(a_{2}+\frac{a_{4}}{2}\right) \int f|\eta|^{2} d x-\frac{a_{4}}{2} \int f^{\prime}(x) x|\eta(x)|^{2} d x .
\end{aligned}
$$

Using the formula for $\left(\left.\omega\right|_{M}\right)_{\eta}$ we have

$$
\begin{aligned}
& \omega\left(\frac{2}{\pi}\left(\int f^{\prime}(x) x|\eta(x)|^{2} d x\right) e_{1} \cdot \eta-\frac{1}{\pi}\left(\int f^{\prime}(x)|\eta(x)|^{2} d x\right) e_{2} \cdot \eta\right. \\
& +\frac{1}{\pi}\left(\int f(x)|\eta(x)|^{2} d x+\int f^{\prime}(x) x|\eta(x)|^{2} d x\right) e_{3} \cdot \eta \\
& \left.+\frac{2}{\pi}\left(\int f^{\prime}(x)|\eta(x)|^{2} d x\right) e_{4} \cdot \eta, Y \cdot \eta\right)
\end{aligned}
$$




$$
=\frac{a_{1}}{2} \int f^{\prime}|\eta|^{2} d x+\left(a_{2}+\frac{a_{4}}{2}\right) \int f|\eta|^{2} d x-\frac{a_{4}}{2} \int f^{\prime}(x) x|\eta(x)|^{2} d x .
$$

By the definition of the projection $P$, the conclusion follows.

LEMMA 4.9.

$P\left(-i \varepsilon \Pi\left(b\left(a+\frac{x}{\mu}\right) \eta\right)+2 B e_{1} \cdot \eta-C e_{2} \cdot \eta+(A+B) e_{3} \cdot \eta+2 C e_{4} \cdot \eta\right)=0$.

Proof. Take $f(x)=\varepsilon b\left(a+\frac{x}{\mu}\right)$ in the above lemma.

REMARK 4.10. Lemma 4.9 and equation (4.4) show that

$$
P\left(-Y \eta-i \varepsilon \Pi\left(b\left(a+\frac{x}{\mu}\right) \eta\right)\right)=-X-\frac{\alpha^{2} \mu^{2}}{2} e_{1}+\frac{\alpha^{2} \mu^{2}}{4} e_{3} .
$$

Thus, $X$ is the orthogonal projection on the manifold of solitons of a significant term of the right-hand side of the equation (4.3) satisfied by $w$.

In the following we intend to give an estimate for $\|X\|$. We need the following definition and Lemma that we cite from [8, Lemma 2.2].

Let $f \in C^{\infty}\left(H_{+}^{\frac{1}{2}}, \mathbb{R}\right)$ and suppose $d f\left(\rho_{0}\right)=0$. Then the Hessian of $f$ at $\rho_{0}$ is well defined $f^{\prime \prime}\left(\rho_{0}\right): T_{\rho_{0}} H_{+}^{\frac{1}{2}} \rightarrow T_{\rho_{0}}^{*} H_{+}^{\frac{1}{2}}$. We identify $T_{\rho_{0}} H_{+}^{\frac{1}{2}}$ and $T_{\rho_{0}}^{*} H_{+}^{\frac{1}{2}}$ using the inner product and we define the Hamiltonian map $F: T_{\rho_{0}} H_{+}^{\frac{1}{2}} \rightarrow T_{\rho_{0}} H_{+}^{\frac{1}{2}}$ by

$$
F=-i f^{\prime \prime}\left(\rho_{0}\right), \quad\left\langle f^{\prime \prime}\left(\rho_{0}\right) X, Y\right\rangle=\omega(Y, F X) .
$$

LEMMA 4.11. Let $N \subset H_{+}^{\frac{1}{2}}$ be a finite-dimensional symplectic submanifold of $H_{+}^{\frac{1}{2}}$ and let $f \in C^{\infty}\left(H_{+}^{\frac{1}{2}}, \mathbb{R}\right)$ such that

$$
X_{f}(\rho) \in T_{\rho} N \subset T_{\rho} H_{+}^{\frac{1}{2}}, \rho \in N .
$$

If $\rho_{0} \in N$ and $d f\left(\rho_{0}\right)=0$, then the Hamiltonian map satisfies

$$
F\left(T_{\rho} N\right) \subset T_{\rho} N .
$$

LEMMA 4.12. If the solution of the perturbed Szegö equation (1.7) can be reparametrized as in Lemma 4.1, $u(t)=g(t) \cdot(\eta+w(t))$, for all $t$ in an interval $\left(t_{1}, t_{2}\right),\|w(t)\|_{L^{2}}$ is small enough, and $\frac{\mu_{0}}{2} \leq \mu(t) \leq \frac{3 \mu_{0}}{2}$, then the vector $X$ defined by

$$
X=\left(\dot{a} \mu-\frac{\alpha^{2} \mu^{2}}{2}+2 B\right) e_{1}+\left(\frac{\dot{\alpha}}{\alpha}-C\right) e_{2}+\left(\dot{\phi}+\frac{\alpha^{2} \mu^{2}}{4}+A+B\right) e_{3}+\left(\frac{\dot{\mu}}{\mu}+2 C\right) e_{4},
$$

where the expressions of $A, B, C$ can be found in equation (4.2), satisfies the inequality

$$
\|X\| \leq C\left(\varepsilon\|w\|_{L^{2}}+\|w\|_{H_{+}^{\frac{1}{2}}}^{2}+\|w\|_{H_{+}^{\frac{1}{2}}}^{3}\right)
$$

REMARK 4.13. Lemma 4.12 yields that if $\|w\|_{H_{+}^{1 / 2}}$ is small, then $\|X\|$ is also small. On the other hand, we noticed in Remark 4.5 that $\|X\|$ measures how far $a, \alpha, \phi, \mu$ are from the effective dynamics (1.9). Thus, the Lemma 4.12 shows that if one can prove that $w$, the part of the flow which is orthogonal to the manifold of solitons, is small, then $a, \alpha, \phi, \mu$ are perturbations of the effective dynamics. 
Proof. Note first that $P(Y \cdot \eta)=Y$, for all $Y \in \mathfrak{g}$.

Since $\omega(w, Y \cdot \eta)=0$, for all $Y \in \mathfrak{g}$, it follows that $P w=0$ and $P \partial_{t} w=\partial_{t} P w=$ 0. Then, by Proposition 4.4 and Lemma 4.9, we have

$$
\begin{aligned}
0= & -X-P(X w)+\alpha^{2} \mu^{2} P(i \mathcal{L} w)-\alpha^{2} \mu^{2} P(i \mathcal{N} w) \\
& +P\left(-i \varepsilon \Pi\left(b\left(a+\frac{x}{\mu}\right) w\right)+2 B e_{1} \cdot w-C e_{2} \cdot w+(A+B) e_{3} \cdot w+2 C e_{4} \cdot w\right) .
\end{aligned}
$$

By Lemma 4.7, we have that

$$
\begin{aligned}
& \|P(X w)\| \leq c\|X\|\|w\|_{L^{2}}, \\
& \|P(i \mathcal{N} w)\| \leq c\|w\|_{H_{+}^{\frac{1}{2}}}^{2}\left(\|w\|_{H_{+}^{\frac{1}{2}}}+1\right) .
\end{aligned}
$$

We prove that $P(-i \mathcal{L} w)=0$. For $\mathcal{E}$ defined by equation $(1.12)$, we have that $X_{\mathcal{E}}$ is tangent to $M$, which corresponds to the fact that if the initial data is in $M$, then the flow of $H_{0}$ stays in $M$. Then,

$$
\left(X_{\mathcal{E}}\right)_{g \cdot \eta} \subset T_{g \cdot \eta} M \subset T_{g \cdot \eta} H_{+}^{\frac{1}{2}} .
$$

Then, by Lemma 4.11, we have that the Hamiltonian map of $\mathcal{E},-i \mathcal{L}$, satisfies

$$
(-i \mathcal{L})\left(T_{\eta} M\right) \subset T_{\eta} M
$$

Then, since $w$ is orthogonal to $T_{\eta} M=\mathfrak{g} \cdot \eta$ and $T_{|\eta|^{2}}, H_{\eta^{2}}$ are symmetric with respect to the real scalar product, we obtain that

$$
\begin{aligned}
\omega(-i \mathcal{L} w, X \cdot \eta) & =\operatorname{Im} \int-i \mathcal{L} w \overline{X \cdot \eta} d x=-\operatorname{Re} \int \mathcal{L} w \overline{X \cdot \eta} d x=-\langle\mathcal{L} w, X \cdot \eta\rangle \\
& =-\langle w, \mathcal{L}(X \cdot \eta)\rangle=\operatorname{Im} \int \overline{w-i \mathcal{L}(X \cdot \eta)} d x=\omega(w,(-i \mathcal{L})(X \cdot \eta))=0 .
\end{aligned}
$$

For the last term, we first notice that we have

$$
\begin{aligned}
& |A|=\left.\frac{\varepsilon}{\pi}\left|\int b\left(a+\frac{x}{\mu}\right)\right| \eta(x)\right|^{2} d x \mid \leq c \varepsilon\|b\|_{L^{\infty}}\|\eta\|_{L^{2}}^{2} \leq c \varepsilon, \\
& |B|=\left.\frac{\varepsilon}{\pi}\left|\int b^{\prime}\left(a+\frac{x}{\mu}\right) x\right| \eta(x)\right|^{2} \frac{d x}{\mu} \mid \leq c \varepsilon\left\|b^{\prime}\right\|_{L^{1}}\left\|x \eta^{2}(x)\right\|_{L^{\infty}} \leq c \varepsilon, \\
& |C|=\left.\frac{\varepsilon}{\pi}\left|\int b^{\prime}\left(a+\frac{x}{\mu}\right)\right| \eta(x)\right|^{2} \frac{d x}{\mu} \mid \leq c \varepsilon\left\|b^{\prime}\right\|_{L^{1}}\|\eta\|_{L^{\infty}}^{2} \leq c \varepsilon .
\end{aligned}
$$

Using the expression of $P$ we found in the proof of Lemma 4.7, we obtain that

$$
\begin{aligned}
& \left\|P\left(-i \varepsilon \Pi\left(b\left(a+\frac{x}{\mu}\right) w\right)+2 B e_{1} \cdot w-C e_{2} \cdot w+(A+B) e_{3} \cdot w+2 C e_{4} \cdot w\right)\right\| \\
& \leq c \varepsilon\|w\|_{L^{2}} .
\end{aligned}
$$

By Lemma 4.6 we have that $\alpha^{2} \mu \leq \alpha_{0}^{2} \mu_{0}$, and thus we have

$$
\|X\| \leq c\left(\|X\|\|w\|_{L^{2}}+\mu\|w\|_{H_{+}^{\frac{1}{2}}}^{2}+\mu\|w\|_{H_{+}^{\frac{1}{2}}}^{3}\right)+c \varepsilon\|w\|_{L^{2}} .
$$

If $\|w\|_{L^{2}}$ is small enough so that $c\|w\|_{L^{2}}<1$, then we write

$$
\left(1-c\|w\|_{L^{2}}\right)\|X\| \leq c\left(\varepsilon\|w\|_{L^{2}}+\mu\|w\|_{H_{+}^{\frac{1}{2}}}^{2}+\mu\|w\|_{H_{+}^{\frac{1}{2}}}^{3}\right)
$$

To conclude, we use the fact that $\mu(t) \leq \frac{3 \mu_{0}}{2}$. 


\section{Coerciveness of the linearized operator $\mathcal{L}$}

In this section we prove that the linearized operator $\mathcal{L}$, defined by equation (1.14), is coercive in directions which are symplectically orthogonal to the manifold of solitons $M$.

LEMMA 5.1. For all $f \in \operatorname{Ker}\left(H_{\eta^{2}}\right) \cap H_{+}^{\frac{1}{2}}$, we have that

$$
\langle\mathcal{L}(f), f\rangle \geq \frac{1}{4}\|f\|_{H_{+}^{\frac{1}{2}}}^{2} .
$$

ProOF. Since $\eta(x)=\frac{1}{x+i}$, we have that $\operatorname{Ker}\left(H_{\eta^{2}}\right)=\left(\frac{x-i}{x+i}\right)^{2} L_{+}^{2}$. Let $f \in \operatorname{Ker}\left(H_{\eta^{2}}\right) \cap H_{+}^{\frac{1}{2}}, f=\left(\frac{x-i}{x+i}\right)^{2} h$, where $h \in H_{+}^{\frac{1}{2}}$. Then

$$
T_{|\eta|^{2}} f=\Pi\left(\frac{1}{(x+i)(x-i)}\left(\frac{x-i}{x+i}\right)^{2} h\right)=\Pi\left(\frac{x-i}{(x+i)^{3}} h\right)=\frac{x-i}{(x+i)^{3}} h
$$

and

$$
\begin{aligned}
\mathcal{L}(f) & =-\frac{i}{2} \partial_{x} f-2 T_{|\eta|^{2}} f-H_{\eta^{2}} f+\frac{1}{4} f \\
& =2 \frac{x-i}{(x+i)^{3}} h-\frac{i}{2}\left(\frac{x-i}{x+i}\right)^{2} \partial_{x} h-2 \frac{x-i}{(x+i)^{3}} h+\frac{1}{4}\left(\frac{x-i}{x+i}\right)^{2} h \\
& =\left(\frac{x-i}{x+i}\right)^{2}\left(-\frac{i}{2} \partial_{x} h+\frac{1}{4} h\right),
\end{aligned}
$$

and thus, using $\left|\frac{x-i}{x+i}\right|=1$ and the Plancherel identity, we obtain

$$
\begin{aligned}
\langle\mathcal{L}(f), f\rangle & =\left\langle\left(\frac{x-i}{x+i}\right)^{2}\left(-\frac{i}{2} \partial_{x} h+\frac{1}{4} h\right),\left(\frac{x-i}{x+i}\right)^{2} h\right\rangle=\left\langle-\frac{i}{2} \partial_{x} h+\frac{1}{4} h, h\right\rangle \\
& =\frac{1}{2} \int_{0}^{\infty} \xi|\hat{h}(\xi)| d \xi+\frac{1}{4}\|f\|_{L^{2}}^{2} \geq \frac{1}{4}\|f\|_{H_{+}^{\frac{1}{2}}}^{2}
\end{aligned}
$$

In what follows we need a Kronecker-type theorem characterizing the Hankel operators of finite rank. We state this theorem bellow. For the proof we refer to [13].

THEOREM $5.2([\mathbf{1 3}])$. The Hankel operator $H_{u}$ has finite rank $N$ if and only if $u$ is a rational function which belongs to $\mathcal{M}(N)$, where

$\mathcal{M}(N)=\left\{\frac{A(z)}{B(z)} \in L_{+}^{2} \mid \operatorname{deg}(B)=N, \operatorname{deg}(A) \leq N-1, B(0)=1\right.$, p.g.c.d. $\left.(A, B)=1\right\}$.

Moreover, if $u \in \mathcal{M}(N), u(z)=\frac{A(z)}{B(z)}$, where $B(z)=\prod_{j=1}^{J}\left(z-p_{j}\right)^{m_{j}}$, with $\sum_{j=1}^{J} m_{j}=N$ and $\operatorname{Im} p_{j}<0$ for all $j=1,2, \ldots, J$, then the range of $H_{u}$ is given by

$$
\operatorname{Ran} H_{u}=\operatorname{span}_{\mathbb{C}}\left\{\frac{1}{\left(z-p_{j}\right)^{m}}, 1 \leq m \leq m_{j}\right\}_{j=1}^{J}
$$

Proposition 5.3. If $w \in H_{+}^{\frac{1}{2}}$ is such that $\omega(w, X \cdot \eta)=0$, for all $X \in \mathfrak{g}$, then

$$
\langle\mathcal{L} w, w\rangle \geq \frac{1}{4}\|w\|_{H_{+}^{\frac{1}{2}}}^{2} .
$$


ProOF. By the Kronecker-type theorem, we have that the range $\operatorname{Ran} H_{\eta^{2}}$ is generated by all the fractions having as a numerator a complex number and as a denominator a factor of $\eta^{2}$. More precisely,

$$
\begin{aligned}
\operatorname{Ran}\left(H_{\eta^{2}}\right) & =\operatorname{span}_{\mathbb{R}}\left\{\frac{1}{x+i}, \frac{i}{x+i}, \frac{1}{(x+i)^{2}}, \frac{i}{(x+i)^{2}}\right\} \\
& =\operatorname{span}_{\mathbb{R}}\left\{\eta, i \eta,-i \partial_{x} \eta, i \partial_{x}(x \eta)\right\} \\
& =\operatorname{span}_{\mathbb{R}}\left\{i e_{1} \cdot \eta, i e_{2} \cdot \eta, i e_{3} \cdot \eta, i e_{4} \cdot \eta\right\} .
\end{aligned}
$$

On the other hand, we have that $\omega(w, X \cdot \eta)=0$ for all $X \in \mathfrak{g}$, which is equivalent to

$$
0=\omega\left(w, e_{j} \cdot \eta\right)=\operatorname{Im} \int w \overline{e_{j} \cdot \eta} d x=\operatorname{Re} \int w \overline{i e_{j} \cdot \eta} d x=\left\langle w, i e_{j} \cdot \eta\right\rangle,
$$

for $j=1,2,3,4$. Thus $w$ belongs to the orthogonal of $\operatorname{Ran}\left(H_{\eta^{2}}\right)$ with respect to the real scalar product. Since $H_{\eta^{2}}$ is $\mathbb{C}$-antilinear, $w$ belongs also to the orthogonal with respect to the Hermitian inner product in $L^{2}$, which is $\operatorname{Ker}\left(H_{\eta^{2}}\right)$. Hence $w \in \operatorname{Ker}\left(H_{\eta^{2}}\right) \cap H_{+}^{\frac{1}{2}}$. By Lemma 5.1, the conclusion then follows.

\section{Main estimates}

In this section we estimate $w$, the part of the flow which is symplectically orthogonal to the manifold of solitons, and prove that it is small.

LEMMA 6.1. If the solution of the perturbed Szegö equation (1.7) can be reparametrized as in Lemma 4.1, $u(t)=g(t) \cdot(\eta+w(t))$ on a time interval $\left(t_{1}, t_{2}\right)$, $\frac{\mu_{0}}{2} \leq \mu(t) \leq \frac{\mu_{0}}{2}$, and $w(t)$ is small enough in the $H_{+}^{\frac{1}{2}}$-norm, then the following estimate holds

$$
\frac{1}{2}\left|\partial_{t}\langle\mathcal{L} w, w\rangle\right| \leq c \varepsilon\|w\|_{H_{+}^{\frac{1}{2}}}+c \varepsilon\|w\|_{H_{+}^{\frac{1}{2}}}^{2}+c\|w\|_{H_{+}^{\frac{1}{2}}}^{3}+c\|w\|_{H_{+}^{\frac{1}{2}}}^{5}
$$

where $c$ is a constant depending on $\alpha_{0}$ and $\mu_{0}$.

Proof. We have that

$$
\begin{aligned}
& \frac{1}{2} \partial_{t}\langle\mathcal{L} w, w\rangle=\left\langle\mathcal{L} w, \partial_{t} w\right\rangle \\
&=\langle\mathcal{L} w,-X \eta\rangle \\
&+\left\langle\mathcal{L} w,-i \varepsilon \Pi\left(b\left(a+\frac{x}{\mu}\right) \eta\right)+2 B e_{1} \cdot \eta-C e_{2} \cdot \eta+(A+B) e_{3} \cdot \eta+2 C e_{4} \cdot \eta\right\rangle \\
&+\langle\mathcal{L} w,-X w\rangle \\
&+\left\langle\mathcal{L} w,-i \varepsilon \Pi\left(b\left(a+\frac{x}{\mu}\right) w\right)+2 B e_{1} \cdot w-C e_{2} \cdot w\right. \\
&\left.\left.+(A+B) e_{3} \cdot w+2 C e_{4} \cdot w\right)\right\rangle \\
&+\left\langle\mathcal{L} w, i \alpha^{2} \mu^{2} \mathcal{L} w\right\rangle-\left\langle\mathcal{L} w, i \alpha^{2} \mu^{2} \mathcal{N} w\right\rangle \\
&= \mathrm{I}+\mathrm{II}+\mathrm{III}+\mathrm{IV}+\mathrm{V}+\mathrm{VI}
\end{aligned}
$$

and we will estimate each of the six terms. The challenge is to deal with the terms containing $\partial_{x} w$ since we only have $w \in H_{+}^{\frac{1}{2}}(\mathbb{R})$. In what follows we focus on such terms, the rest of the terms being easier to handle. 
We set $X=\sum_{j=1}^{4} a_{j} e_{j}$. By Lemma 4.12, we have that

$$
\left|a_{j}\right| \leq c\left(\varepsilon\|w\|_{L^{2}}+\|w\|_{H_{+}^{\frac{1}{2}}}^{2}+\|w\|_{H_{+}^{\frac{1}{2}}}^{3} .\right.
$$

For I, we integrate by parts

$$
\operatorname{Re}\left(\bar{a}_{1} \int \frac{i}{2} \partial_{x} w \overline{e_{1} \cdot \eta} d x\right)=\operatorname{Re}\left(\bar{a}_{1} \int \frac{i}{2} w \partial_{x}^{2} \bar{\eta} d x\right)
$$

and apply the Cauchy-Schwarz inequality for each term. We obtain

$$
|\mathrm{I}| \leq c\|X\|\|w\|_{L^{2}} \leq c\left(\varepsilon\|w\|_{L^{2}}^{2}+\|w\|_{H_{+}^{\frac{1}{2}}}^{3}+\|w\|_{H_{+}^{\frac{1}{2}}}^{4}\right) .
$$

For II, integrating by parts and using Cauchy-Schwarz, we have

$$
\begin{aligned}
\operatorname{Re}\left(\int \frac{i}{2} \partial_{x} w \cdot \overline{i \varepsilon b\left(a+\frac{x}{\mu}\right)} \bar{\eta} d x\right) & \\
& =\frac{\varepsilon}{2} \operatorname{Re}\left(\int w \overline{b^{\prime}\left(a+\frac{x}{\mu}\right)} \frac{1}{\mu} \bar{\eta} d x+\int \overline{w b\left(a+\frac{x}{\mu}\right)} \bar{\eta}^{\prime} d x\right) \\
& \leq c \varepsilon\|w\|_{L^{2}}\|\eta\|_{L^{\infty}}\left\|b^{\prime}\right\|_{L^{2}} \frac{\mu^{1 / 2}}{\mu}+c \varepsilon\|w\|_{L^{2}}\left\|\eta^{\prime}\right\|_{L^{2}}\|b\|_{L^{\infty}} \\
& \leq c \varepsilon\left(1+\frac{1}{\mu^{1 / 2}}\right)\|w\|_{L^{2}} .
\end{aligned}
$$

Using the equation (4.5) for the rest of the terms, we obtain

$$
|\mathrm{II}| \leq c \varepsilon\|w\|_{L^{2}}
$$

For III and IV we analyze each term. Besides integrating by parts and using Cauchy-Schwarz or Hölder inequalities, a key ingredient is the fact that we deal with the real scalar product.

$$
\begin{aligned}
\mathrm{III} & =\langle\mathcal{L} w,-X w\rangle=\operatorname{Re}\left(\sum_{j=1}^{4} a_{j} \int \frac{i}{2} \partial_{x} w \overline{e_{j} \cdot w} d x+2 \sum_{j=1}^{4} a_{j} \int|\eta|^{2} w \overline{e_{j} \cdot w} d x\right. \\
& \left.+\sum_{j=1}^{4} a_{j} \int \eta^{2} \overline{w e_{j} \cdot w} d x-\frac{1}{4} \sum_{j=1}^{4} a_{j} \int w \overline{e_{j} \cdot w} d x\right)=(\mathrm{i})+(\mathrm{ii})+(\mathrm{iii})+(\mathrm{iv}) .
\end{aligned}
$$

Then

$$
\begin{aligned}
(\mathrm{i})= & \operatorname{Re}\left(-a_{1} \frac{i}{2} \int\left|\partial_{x} w\right|^{2}+a_{2} \frac{i}{2} \int \partial_{x} w \bar{w} d x+\frac{a_{3}}{4} \int \partial_{x}\left(|w|^{2}\right) d x\right. \\
& \left.\quad+a_{4} \frac{i}{2} \int \partial_{x} w \bar{w} d x+a_{4} \frac{i}{2} \int x\left|\partial_{x} w\right|^{2} d x\right) \\
= & -\frac{a_{2}+a_{4}}{2} \int \frac{1}{i} \partial_{x} w \bar{w} d x=-\frac{a_{2}+a_{4}}{2} \int_{0}^{\infty} \xi|\hat{w}(\xi)|^{2} d \xi \\
= & -\frac{a_{2}+a_{4}}{2}\|w\|_{\dot{H}_{+}^{1 / 2}}^{2} \leq\|X\|\|w\|_{H_{+}^{\frac{1}{2}}}^{2}
\end{aligned}
$$


by the Hölder inequality we have

$$
\begin{aligned}
&(\mathrm{ii})= 2 \operatorname{Re}\left(-a_{1} \int|\eta|^{2} w \partial_{x} \bar{w} d x+a_{2} \int|\eta|^{2}|w|^{2} d x-a_{3} i \int|\eta|^{2}|w|^{2} d x\right. \\
&\left.\quad+a_{4} \int|\eta|^{2}|w|^{2} d x+a_{4} \int|\eta|^{2} x w \partial_{x} \bar{w} d x\right) \\
&=-a_{1} \int|\eta|^{2} \partial_{x}\left(|w|^{2}\right) d x+2\left(a_{2}+a_{4}\right) \int|\eta|^{2}|w|^{2} d x+a_{4} \int|\eta|^{2} x \partial_{x}\left(|w|^{2}\right) d x \\
& \leq c\|X\|\|w\|_{L^{2}}^{2}
\end{aligned}
$$

similarly

$$
\begin{aligned}
&(\mathrm{iii})= \operatorname{Re}\left(-\frac{a_{1}}{2} \int \eta^{2} \partial_{x}\left(\bar{w}^{2}\right) d x+a_{2} \int \eta^{2} \bar{w}^{2} d x-a_{3} i \int \eta^{2} \bar{w}^{2} d x\right. \\
&\left.+a_{4} \int \eta^{2} \bar{w}^{2} d x+\frac{a_{4}}{2} \int \eta^{2} x \partial_{x}\left(\bar{w}^{2}\right) d x\right) \\
& \leq c\|X\|\|w\|_{L^{2}}^{2}
\end{aligned}
$$

and

$$
\begin{aligned}
\text { (iv) }=-\frac{1}{4} \operatorname{Re}\left(-a_{1} \int w \partial_{x} \bar{w} d x+a_{2} \int|w|^{2} d x-a_{3} i \int|w|^{2} d x\right. \\
\left.\quad+a_{4} \int|w|^{2} d x+\frac{a_{4}}{2} \int x w \partial_{x} \bar{w} d x\right) \\
=-\frac{1}{4}\left(-\frac{a_{1}}{2} \int \partial_{x}\left(|w|^{2}\right) d x+\left(a_{2}+a_{4}\right) \int|w|^{2} d x+\frac{a_{4}}{2} \int x \partial_{x}\left(|w|^{2}\right) d x\right) \\
=-\frac{1}{4}\left(a_{2}+\frac{a_{4}}{2}\right) \int|w|^{2} d x \leq c\|X\|\|w\|_{L^{2}}^{2} .
\end{aligned}
$$

Hence

$$
|\mathrm{III}| \leq c\|X\|\|w\|_{H_{+}^{\frac{1}{2}}}^{2} \leq c\left(\varepsilon\|w\|_{H_{+}^{\frac{1}{2}}}^{3}+\|w\|_{H_{+}^{\frac{1}{2}}}^{4}+\|w\|_{H_{+}^{\frac{1}{2}}}^{5}\right) .
$$

For IV we have

$$
\begin{aligned}
& \mathrm{IV}=\operatorname{Re} \int \frac{i}{2} \partial_{x} w\left(i \varepsilon b\left(a+\frac{x}{\mu}\right) \bar{w}+2 B \overline{e_{1} \cdot w}-C \overline{e_{2} \cdot w}\right. \\
& \left.+(A+B) \overline{e_{3} \cdot w}+2 C \overline{e_{4} \cdot w}\right) d x \\
& +2 \operatorname{Re} \int|\eta|^{2} w\left(i \varepsilon \overline{\Pi\left(b\left(a+\frac{x}{\mu}\right) w\right)}+2 B \overline{\overline{e_{1} \cdot w}}-C \overline{\bar{e}_{2} \cdot w}\right. \\
& +\left(A+B \overline{e_{3} \cdot w}+2 C \overline{e_{4} \cdot w}\right) d x \\
& +\operatorname{Re} \int \eta^{2} \bar{w}\left(i \varepsilon \overline{\Pi\left(b\left(a+\frac{x}{\mu}\right) w\right)}+2 B \overline{e_{1} \cdot w}-C \overline{e_{2} \cdot w}\right. \\
& \left.+(A+B) \overline{e_{3} \cdot w}+2 C \overline{e_{4} \cdot w}\right) d x \\
& -\frac{1}{4} \operatorname{Re} \int w\left(i \varepsilon b\left(a+\frac{x}{\mu}\right) \bar{w}+2 B \overline{e_{1} \cdot w}-C \overline{e_{2} \cdot w}\right. \\
& \left.+(A+B) \overline{e_{3} \cdot w}+2 C \overline{e_{4} \cdot w}\right) d x \\
& =(\mathrm{i})+(\mathrm{ii})+(\mathrm{iii})+(\mathrm{iv}) .
\end{aligned}
$$


By the equations (4.5) and the Sobolev embedding $H^{\frac{1}{2}}(\mathbb{R}) \subset L^{p}(\mathbb{R}), 2 \leq p<\infty$, we have

$$
\begin{aligned}
(\mathrm{i})= & -\frac{1}{4} \int \varepsilon b\left(a+\frac{x}{\mu}\right) \partial_{x}\left(|w|^{2}\right) d x-B \operatorname{Re} \int i\left|\partial_{x} w\right|^{2} d x-\frac{C}{2} \operatorname{Re} \int i \partial_{x} w \bar{w} d x \\
& +\frac{A+B}{4} \int \partial_{x}\left(|w|^{2}\right) d x+C \operatorname{Re} \int i \partial_{x} w \bar{w} d x \\
= & \frac{\varepsilon}{4 \mu} \int b^{\prime}\left(a+\frac{x}{\mu}\right)|w|^{2} d x-\frac{C}{2}\|w\|_{\dot{H}_{+}^{\frac{1}{2}}}^{2} \leq \frac{c \varepsilon}{\mu^{1 / 2}}\left\|b^{\prime}\right\|_{L^{2}}\|w\|_{L^{4}}^{2}+c \varepsilon\|w\|_{\dot{H}_{+}^{\frac{1}{2}}}^{2} \\
\leq & c \varepsilon\left(1+\frac{1}{\mu^{1 / 2}}\right)\|w\|_{H_{+}^{\frac{1}{2}}}^{2},
\end{aligned}
$$

For (ii) we only analyze the terms containing $\partial_{x} w$. By the equations (4.5), we obtain

$$
\begin{aligned}
& -4 B \operatorname{Re} \int|\eta|^{2} w \partial_{x} \bar{w} d x+4 C \operatorname{Re} \int|\eta|^{2} x w \partial_{x} \bar{w} d x \\
& =-2 B \int|\eta|^{2} \partial_{x}\left(|w|^{2}\right) d x+2 C \int|\eta|^{2} x \partial_{x}\left(|w|^{2}\right) d x \\
& =2 B \int \partial_{x}\left(|\eta|^{2}\right)|w|^{2} d x-2 C \int \partial_{x}\left(|\eta|^{2} x\right)|w|^{2} d x \\
& \leq c \varepsilon\|w\|_{L^{2}}^{2} .
\end{aligned}
$$

Thus

$$
\text { (ii) } \leq c \varepsilon\left(1+\frac{1}{\mu^{1 / 2}}\right)\|w\|_{L^{2}}^{2}
$$

and similarly we obtain the same bound for (iii). Computing the last term, we obtain that $(\mathrm{iv})=0$. Hence

$$
|\mathrm{IV}| \leq c \varepsilon\left(1+\frac{1}{\mu^{1 / 2}}\right)\|w\|_{H_{+}^{\frac{1}{2}}}^{2} .
$$

Since we work with the real scalar product, it follows immediately that $\mathrm{V}=0$. For VI again we only analyze the terms containing $\partial_{x} w$. The important step is to group together $w \bar{\eta}+\bar{w} \eta \in \mathbb{R}$.

$$
\begin{aligned}
& -\alpha^{2} \mu^{2}\left\langle\frac{i}{2} \partial_{x} w, i\left(|w|^{2} w+2|w|^{2} \eta+w^{2} \bar{\eta}\right)\right\rangle \\
& \left.=-\alpha^{2} \mu^{2}\left(\frac{1}{4} \int|w|^{2} \partial_{x}\left(|w|^{2}\right)\right) d x+\frac{1}{2} \operatorname{Re} \int|w|^{2} \partial_{x} w \bar{\eta} d x+\frac{1}{2} \operatorname{Re} \int \partial_{x} w \bar{w}(w \bar{\eta}+\bar{w} \eta) d x\right) \\
& =-\alpha^{2} \mu^{2}\left(\frac{1}{8} \int \partial_{x}\left(|w|^{4}\right) d x+\frac{1}{2} \operatorname{Re} \int|w|^{2} \partial_{x} w \bar{\eta} d x+\frac{1}{2} \int \partial_{x}\left(|w|^{2}\right) 2 \operatorname{Re}(w \bar{\eta}) d x\right) \\
& =-\alpha^{2} \mu^{2}\left(\frac{1}{2} \operatorname{Re} \int|w|^{2} \partial_{x} w \bar{\eta} d x-\frac{1}{2} \int|w|^{2} 2 \operatorname{Re}\left(\bar{\eta} \partial_{x} w+w \partial_{x} \bar{\eta}\right) d x\right) \\
& =\alpha^{2} \mu^{2} \operatorname{Re} \int|w|^{2} w \partial_{x} \bar{\eta} d x \leq c \alpha^{2} \mu^{2}\|w\|_{H_{+}^{\frac{1}{2}}}^{3}
\end{aligned}
$$

For the other terms it is enough to apply the Cauchy-Schwarz and Sobolev inequalities. Using Lemma 4.6 we obtain

$$
|\mathrm{VI}| \leq c \alpha^{2} \mu^{2}\left(\|w\|_{H_{+}^{\frac{1}{2}}}^{3}+\|w\|_{H_{+}^{\frac{1}{2}}}^{4}\right) \leq c \alpha_{0}^{2} \mu_{0} \mu\left(\|w\|_{H_{+}^{\frac{1}{2}}}^{3}+\|w\|_{H_{+}^{\frac{1}{2}}}^{4}\right) .
$$


In the following, we combine the inequality in Lemma 6.1 with the coerciveness properties of the linearized operator $\mathcal{L}$, to obtain an estimate for $\|w\|_{H_{+}^{\frac{1}{2}}}$.

Proposition 6.2. Suppose the solution of the perturbed Szegö equation (1.7) can be reparametrized as in Lemma 4.1, $u(t)=g(t) \cdot(\eta+w(t))$ on a time interval $\left[t_{1}, t_{2}\right]$ and $\frac{\mu_{0}}{2} \leq \mu(t) \leq \frac{\mu_{0}}{2}$. Let $0<\varepsilon \ll 1$ and $0<\delta<\frac{1}{2}$. If $\left|t_{2}-t_{1}\right| \leq \frac{1}{\varepsilon^{\frac{1}{2}-\delta}}$ and

$$
\|w\|_{L^{\infty}\left(\left[t_{1}, t_{2}\right], H_{+}^{\frac{1}{2}}\right)} \leq \varepsilon^{\frac{1}{2}}
$$

then

$$
\|w\|_{L^{\infty}\left(\left[t_{1}, t_{2}\right], H_{+}^{\frac{1}{2}}\right)} \leq c_{0}\left\|w\left(t_{1}\right)\right\|_{H_{+}^{\frac{1}{2}}}+c_{0} \varepsilon^{\frac{1+\delta}{2}}
$$

where $c_{0}>2$ is a constant depending only on $\alpha_{0}$ and $\mu_{0}$.

PROOF. Integrating from $t_{1}$ to $t_{2}$ the estimate in Lemma 6.1, we have that

$$
\begin{aligned}
\left\langle\mathcal{L} w\left(t_{2}\right), w\left(t_{2}\right)\right\rangle \leq & \left\langle\mathcal{L} w\left(t_{1}\right), w\left(t_{1}\right)\right\rangle+c\left(t_{2}-t_{1}\right) \varepsilon\|w\|_{L^{\infty}\left(\left[t_{1}, t_{2}\right], H_{+}^{\frac{1}{2}}\right)} \\
& +c\left(t_{2}-t_{1}\right) \varepsilon\|w\|_{L^{\infty}\left(\left[t_{1}, t_{2}\right], H_{+}^{\frac{1}{2}}\right)}^{2}+c\left(t_{2}-t_{1}\right)\|w\|_{L^{\infty}\left(\left[t_{1}, t_{2}\right], H_{+}^{\frac{1}{2}}\right)}^{3} \\
& +c\left(t_{2}-t_{1}\right)\|w\|_{L^{\infty}\left(\left[t_{1}, t_{2}\right], H_{+}^{\frac{1}{2}}\right)}^{4}+\left(t_{2}-t_{1}\right)\|w\|_{L_{\left(\left[t_{1}, t_{2}\right], H_{+}^{\frac{1}{2}}\right)}^{5}}^{4}
\end{aligned}
$$

On the other hand, we have

$$
\begin{aligned}
\left\langle\mathcal{L} w\left(t_{1}\right), w\left(t_{1}\right)\right\rangle= & \frac{1}{2} \operatorname{Re} \int \frac{1}{i} \partial_{x} w\left(t_{1}\right) \bar{w}\left(t_{1}\right) d x-2 \int|\eta|^{2}\left|w\left(t_{1}\right)\right|^{2} d x \\
& -\operatorname{Re} \int \eta^{2} \bar{w}\left(t_{1}\right)^{2} d x+\frac{1}{4} \int\left|w\left(t_{1}\right)\right|^{2} d x \\
\leq & \frac{1}{2}\left\|w\left(t_{1}\right)\right\|_{H_{+}^{\frac{1}{2}}}^{2}+2\|\eta\|_{L^{\infty}}^{2}\left\|w\left(t_{1}\right)\right\|_{L^{2}}^{2}+\|\eta\|_{L^{\infty}}^{2}\left\|w\left(t_{1}\right)\right\|_{L^{2}}^{2}+\frac{1}{4}\left\|w\left(t_{1}\right)\right\|_{L^{2}}^{2} \\
\leq & 4\left\|w\left(t_{1}\right)\right\|_{H_{+}^{\frac{1}{2}}}^{2}
\end{aligned}
$$

Together with the coerciveness of the linearized operator $\mathcal{L}$ in Proposition 5.3, this yields

$$
\begin{aligned}
\frac{1}{4}\|w\|_{L^{\infty}\left(\left[t_{1}, t_{2}\right], H_{+}^{\frac{1}{2}}\right)}^{2} \leq & 4\left\|w\left(t_{1}\right)\right\|_{H_{+}^{\frac{1}{2}}}^{2}+c\left(t_{2}-t_{1}\right) \varepsilon\|w\|_{L^{\infty}\left(\left[t_{1}, t_{2}\right], H_{+}^{\frac{1}{2}}\right)} \\
& +c\left(t_{2}-t_{1}\right) \varepsilon\|w\|_{L^{\infty}\left(\left[t_{1}, t_{2}\right], H_{+}^{\frac{1}{2}}\right)}^{2}+c\left(t_{2}-t_{1}\right)\|w\|_{L^{\infty}\left(\left[t_{1}, t_{2}\right], H_{+}^{\frac{1}{2}}\right)}^{3} \\
& +c\left(t_{2}-t_{1}\right)\|w\|_{L^{\infty}\left(\left[t_{1}, t_{2}\right], H_{+}^{\frac{1}{2}}\right)}^{5} \cdot
\end{aligned}
$$

Since $c\left(t_{2}-t_{1}\right) \varepsilon=c \varepsilon^{\frac{1}{2}+\delta}<\frac{1}{8}$ we can pass the term $c\left(t_{2}-t_{1}\right) \varepsilon\|w\|_{H_{+}^{\frac{1}{2}}}^{2}$ to the left hand-side of the inequality and with the estimates in the hypothesis we obtain

$$
\frac{1}{8}\|w\|_{L^{\infty}\left(\left[t_{1}, t_{2}\right], H_{+}^{\frac{1}{2}}\right)}^{2} \leq 4\left\|w\left(t_{1}\right)\right\|_{H_{+}^{\frac{1}{2}}}^{2}+3 c \varepsilon^{1+\delta} .
$$

This gives us the conclusion with the constant $c_{0}=\max (32,24 c)$ depending only on $\alpha_{0}, \mu_{0}$.

The proposition below is the main step in proving Theorem 1.1. 
Proposition 6.3. Let $\Sigma$ be a compact subset of $\mathbb{R} \times \mathbb{R}_{+}^{*} \times \mathbb{T} \times \mathbb{R}_{+}^{*}, 0<\delta<\frac{1}{2}$, and let $\varepsilon>0$ be such that $\varepsilon^{\frac{1}{2}}<\gamma_{0}$, where $\gamma_{0}$ was defined in Lemma 4.1. Suppose $\inf _{g \in \Sigma}\|u(0)-g \cdot \eta\|_{H_{+}^{\frac{1}{2}}} \leq \varepsilon^{\frac{1}{2}+\frac{\delta}{2}}$. Then, for all

$$
0<t \leq \frac{\delta}{6 \ln c_{0}} \cdot \frac{1}{\varepsilon^{\frac{1}{2}-\delta}} \ln \left(\frac{1}{\varepsilon}\right),
$$

the solution of the perturbed Szegö equation (1.7) at time $t$ can be parameterized as in Lemma 4.1, $u(t)=g(t)(\eta+w(t))$. Moreover, we have

$$
\|w\|_{L^{\infty}\left([0, t], H_{+}^{\frac{1}{2}}\right)} \leq \varepsilon^{-\frac{\delta}{6}}\|w(0)\|_{H_{+}^{\frac{1}{2}}}+\varepsilon^{\frac{1}{2}+\frac{\delta}{3}}
$$

and

$$
\frac{\mu_{0}}{2} \leq \mu(t) \leq \frac{3 \mu_{0}}{2}
$$

Proof. We use a bootstrap argument. Set

$$
T:=\sup \left\{t>0 \mid \inf _{g \in \Sigma}\|u(t)-g \cdot \eta\|_{L^{\infty}\left([0, t], H_{+}^{1 / 2}\right)} \leq \varepsilon^{\frac{1}{2}}, \frac{\mu_{0}}{2} \leq \mu(t) \leq \frac{3 \mu_{0}}{2}\right\} .
$$

We intend to show that $T \geq \frac{\delta}{6 \ln c_{0}} \cdot \frac{1}{\varepsilon^{\frac{1}{2}-\delta}} \ln \left(\frac{1}{\varepsilon}\right)$. Suppose by contradiction that

$$
T<\frac{\delta}{6 \ln c_{0}} \cdot \frac{1}{\varepsilon^{\frac{1}{2}-\delta}} \ln \left(\frac{1}{\varepsilon}\right) .
$$

Since $\inf _{g \in \Sigma}\|u(t)-g(t) \cdot \eta\|_{L^{\infty}\left([0, t], H_{+}^{1 / 2}\right)} \leq \varepsilon^{\frac{1}{2}}<\gamma_{0}$ for all $0<t<T$, it follows by Lemma 4.1 that the solution of the perturbed Szegö equation (1.7) can be reparametrized as $u(t)=g(t) \cdot(\eta+w(t))$ for all $0<t<T$, and moreover $\|w(t)\|_{L^{\infty}\left([0, t], H_{+}^{1 / 2}\right)} \leq \varepsilon^{\frac{1}{2}}$. We apply the Proposition 6.2 successively on the intervals $\left[0, \frac{1}{\varepsilon^{\frac{1}{2}-\delta}}\right],\left[\frac{1}{\varepsilon^{\frac{1}{2}-\delta}}, \frac{2}{\varepsilon^{\frac{1}{2}-\delta}}\right], \ldots,\left[\frac{k-1}{\varepsilon^{\frac{1}{2}-\delta}}, \frac{k}{\varepsilon^{\frac{1}{2}-\delta}}\right]$. For $t$ in the interval $\left[0, \frac{1}{\varepsilon^{\frac{1}{2}-\delta}}\right]$, we obtain

$$
\|w(t)\|_{H_{+}^{\frac{1}{2}}} \leq c_{0}\|w(0)\|_{H_{+}^{\frac{1}{2}}}+c_{0} \varepsilon^{\frac{1+\delta}{2}} .
$$

Using this information for $t=\frac{1}{\varepsilon^{\frac{1}{2}-\delta}}$, we obtain for $t \in\left[\frac{1}{\varepsilon^{\frac{1}{2}-\delta}}, \frac{2}{\varepsilon^{\frac{1}{2}-\delta}}\right]$ that

$$
\|w(t)\|_{H_{+}^{\frac{1}{2}}} \leq c_{0}^{2}\|w(0)\|_{H_{+}^{\frac{1}{2}}}+c_{0}\left(1+c_{0}\right) \varepsilon^{\frac{1+\delta}{2}} .
$$

Ultimately, we have that for all $t \in\left[0, \frac{k}{\varepsilon^{\frac{1}{2}-\delta}}\right]$

$$
\|w(t)\|_{H_{+}^{\frac{1}{2}}} \leq c_{0}^{k}\|w(0)\|_{H_{+}^{\frac{1}{2}}}+c_{0}\left(\sum_{j=0}^{k-1} c_{0}^{j}\right) \varepsilon^{\frac{1+\delta}{2}}=c_{0}^{k}\|w(0)\|_{H_{+}^{\frac{1}{2}}}+c_{0} \frac{c_{0}^{k}-1}{c_{0}-1} \varepsilon^{\frac{1+\delta}{2}} .
$$

Since $c_{0}>2$, we have that $c_{0} \frac{c_{0}^{k}-1}{c_{0}-1} \leq 2 c_{0}^{k}$. Take $k$ such that $c_{0}^{k}=\varepsilon^{-\frac{\delta}{6}}$, which is equivalent to

Then,

$$
k=\frac{\delta}{6 \ln c_{0}} \ln \left(\frac{1}{\varepsilon}\right) .
$$

$$
\|w\|_{L^{\infty}\left(\left[0, \frac{k}{\varepsilon^{\frac{1}{2}-\delta}}\right], H_{+}^{\frac{1}{2}}\right)} \leq \varepsilon^{-\frac{\delta}{6}}\|w(0)\|_{H_{+}^{\frac{1}{2}}}+2 \varepsilon^{\frac{1}{2}+\frac{\delta}{3}} \leq 3 \varepsilon^{\frac{1}{2}+\frac{\delta}{3}}
$$


Therefore, we have for $0 \leq t \leq \frac{\delta}{6 \ln c_{0}} \cdot \frac{1}{\varepsilon^{\frac{1}{2}-\delta}} \ln \left(\frac{1}{\varepsilon}\right)$ that

$$
\|w(t)\|_{L^{\infty}\left([0, t], H_{+}^{\frac{1}{2}}\right)} \leq 3 \varepsilon^{\frac{1}{2}+\frac{\delta}{3}}
$$

and by Lemma 4.12 it follows that

$$
\|X\| \leq c \varepsilon^{1+\frac{2 \delta}{3}} .
$$

By the definition of $X(4.2)$, it follows that

$$
\left|\frac{\dot{\mu}}{\mu}+2 C\right| \leq c \varepsilon^{1+\frac{2 \delta}{3}}
$$

Thus

$$
\frac{\dot{\mu}}{\mu} \leq-\frac{2 \varepsilon}{\pi} \int b^{\prime}\left(a+\frac{x}{\mu}\right)|\eta(x)|^{2} \frac{d x}{\mu}+c \varepsilon^{1+\frac{2 \delta}{3}} .
$$

Integrating from 0 to $t$, where $0 \leq t \leq \frac{\delta}{6 \ln c_{0}} \cdot \frac{1}{\varepsilon^{\frac{1}{2}-\delta}} \ln \left(\frac{1}{\varepsilon}\right)$, and using the change of variables $y=a+\frac{x}{\mu}$, we obtain that

$$
\ln \left(\frac{\mu(t)}{\mu_{0}}\right) \leq c\left(\varepsilon\left\|b^{\prime}\right\|_{L^{1}}\|\eta\|_{L^{\infty}}^{2}+\varepsilon^{1+\frac{2 \delta}{3}}\right) t \leq \frac{c \delta}{6 \ln c_{0}} \cdot \varepsilon^{\frac{1}{2}+\delta} \ln \left(\frac{1}{\varepsilon}\right) .
$$

Since around zero we have the Taylor expansion $\ln (1+x)=x+O\left(x^{2}\right)$, it follows that

Hence, we obtain

$$
\frac{\mu(t)-\mu_{0}}{\mu_{0}} \leq \frac{c \delta}{6 \ln c_{0}} \cdot \varepsilon^{\frac{1}{2}+\delta} \ln \left(\frac{1}{\varepsilon}\right) .
$$

$$
\left|\mu(t)-\mu_{0}\right| \leq \tilde{c}_{0} \delta \varepsilon^{\frac{1}{2}+\delta} \ln \left(\frac{1}{\varepsilon}\right),
$$

where $\tilde{c}_{0}$ is a constant depending on $\alpha_{0}, \mu_{0}$. Thus,

$$
\frac{2 \mu_{0}}{3} \leq \mu(t) \leq \frac{4 \mu_{0}}{3} \quad \text { for } \quad 0 \leq t \leq \frac{\delta}{6 \ln c_{0}} \cdot \frac{1}{\varepsilon^{\frac{1}{2}-\delta}} \ln \left(\frac{1}{\varepsilon}\right)
$$

Equations (6.3) and (6.4) show that the conditions in the definition of $T$ (6.2) hold with better bounds, i.e. $3 \varepsilon^{\frac{1}{2}+\frac{\delta}{3}}$ instead of $\varepsilon^{\frac{1}{2}}, \frac{4 \mu_{0}}{3}$ instead of $\frac{3 \mu_{0}}{2}$, and $\frac{2 \mu_{0}}{3}$ instead of $\frac{\mu_{0}}{2}$, for $0 \leq t \leq \frac{\delta}{6 \ln c_{0}} \cdot \frac{1}{\varepsilon^{\frac{1}{2}-\delta}} \ln \left(\frac{1}{\varepsilon}\right)$. Since $w(t)$ and $\mu(t)$ are continuous with respect to time, it follows that there exists $t_{0}>0$ such that the conditions in the definition of $T$ with exactly the same bounds as in that definition hold for times $0 \leq t \leq \frac{\delta}{6 \ln c_{0}} \cdot \frac{1}{\varepsilon^{\frac{1}{2}-\delta}} \ln \left(\frac{1}{\varepsilon}\right)+t_{0}$. This contradicts our assumption $T<$ $\frac{\delta}{6 \ln c_{0}} \cdot \frac{1}{\varepsilon^{\frac{1}{2}-\delta}} \ln \left(\frac{1}{\varepsilon}\right)$. Therefore, the conclusion of the proposition follows.

\section{Proof of Theorem 1.1}

In this section we prove that Theorem 1.1 follows from Proposition 6.3.

Proof of Theorem 1.1. First we notice that $u(0)=g(0) \cdot \eta$, where $g(0)=\left(a_{0}, \alpha_{0}, \phi_{0}, \mu_{0}\right)$. Thus, by Proposition 6.3, it follows that $u(t)$ can be reparametrized as $u(t)=g(t) \cdot(\eta+w(t))$ for times $0 \leq t \leq \frac{\delta}{6 \ln c_{0}} \cdot \frac{1}{\varepsilon^{\frac{1}{2}-\delta}} \ln \left(\frac{1}{\varepsilon}\right)$, and moreover

$$
\|w(t)\|_{L^{\infty}\left([0, t], H_{+}^{\frac{1}{2}}\right)} \leq 3 \varepsilon^{\frac{1}{2}+\frac{\delta}{3}}, \quad \frac{\mu_{0}}{2} \leq \mu(t) \leq \frac{3 \mu_{0}}{2} .
$$


By Lemma 4.12 we then obtain

$$
\|X\| \leq c \varepsilon^{1+\frac{2 \delta}{3}}
$$

Proceeding as in the last part of the proof of Proposition 6.3, we obtain that

$$
\left|\mu(t)-\mu_{0}\right| \leq \tilde{c}_{0} \delta \varepsilon^{\frac{1}{2}+\delta} \ln \left(\frac{1}{\varepsilon}\right) .
$$

for $0 \leq t \leq \frac{\delta}{6 \ln c_{0}} \cdot \frac{1}{\varepsilon^{\frac{1}{2}-\delta}} \ln \left(\frac{1}{\varepsilon}\right)$. Similarly we have $\left|\bar{\mu}(t)-\mu_{0}\right| \leq \tilde{c}_{0} \delta \varepsilon^{\frac{1}{2}+\delta} \ln \left(\frac{1}{\varepsilon}\right)$. Then

$$
|\mu(t)-\bar{\mu}(t)| \leq \tilde{c}_{0} \delta \varepsilon^{\frac{1}{2}+\delta} \ln \left(\frac{1}{\varepsilon}\right) .
$$

By equation (7.1) and using the definition of $X$, it follows that

$$
\left|\frac{\dot{\alpha}}{\alpha}-C\right| \leq c \varepsilon^{1+\frac{2 \delta}{3}}
$$

Thus

$$
\frac{\dot{\alpha}}{\alpha} \leq \frac{\varepsilon}{\pi} \int b^{\prime}\left(a+\frac{x}{\mu}\right)|\eta(x)|^{2} \frac{d x}{\mu}+c \varepsilon^{1+\frac{2 \delta}{3}} .
$$

Proceeding as we did for $\mu(t)$ and possibly making the constant $\tilde{c}_{0}$ larger, we obtain that

$$
\begin{gathered}
\left|\alpha(t)-\alpha_{0}\right| \leq \tilde{c}_{0} \delta \varepsilon^{\frac{1}{2}+\delta} \ln \left(\frac{1}{\varepsilon}\right), \\
|\alpha(t)-\bar{\alpha}(t)| \leq \tilde{c}_{0} \delta \varepsilon^{\frac{1}{2}+\delta} \ln \left(\frac{1}{\varepsilon}\right),
\end{gathered}
$$

for $0 \leq t \leq \frac{\delta}{6 \ln c_{0}} \cdot \frac{1}{\varepsilon^{\frac{1}{2}-\delta}} \ln \left(\frac{1}{\varepsilon}\right)$.

We thus proved that for the above range of time, $\mu(t)$ and $\alpha(t)$ stay close to $\mu_{0}$ and $\alpha_{0}$ respectively. The definition of $X(4.2)$ and the estimate (7.1) then yield that $a, \alpha, \phi, \mu$ satisfy the perturbed effective dynamics (1.8) in the statement of Theorem 1.1.

By Lemma 4.6 we have that $\|w\|_{L^{2}}^{2}=\pi\left(\frac{\alpha_{0}^{2} \mu_{0}}{\alpha^{2} \mu}-1\right)$. Then, the equations satisfied by $\bar{\alpha}$ and $\bar{\mu}$ yield $\partial_{t}\left(\bar{\alpha}^{2} \bar{\mu}\right)=0$, and thus we obtain that

$$
\alpha^{2} \mu=\alpha_{0}^{2} \mu_{0}+c \varepsilon^{1+\frac{2 \delta}{3}}, \quad \bar{\alpha}^{2} \bar{\mu}=\alpha_{0}^{2} \mu_{0}
$$

Subtracting the equations satisfied by $\phi$ and $\bar{\phi}$, we then obtain that

$$
\begin{aligned}
|\dot{\phi}-\dot{\bar{\phi}}| & =\left.\left|-\frac{\alpha_{0}^{2} \mu_{0}}{4}(\mu-\bar{\mu})-\frac{\varepsilon}{\pi} \int\left(b\left(a+\frac{x}{\mu}\right)-b\left(\bar{a}+\frac{x}{\bar{\mu}}\right)\right)\right| \eta\right|^{2} d x \\
& \quad-\frac{\varepsilon}{\pi} \int\left(b^{\prime}\left(a+\frac{x}{\mu}\right)-b^{\prime}\left(\bar{a}+\frac{x}{\bar{\mu}}\right)\right) x|\eta(x)|^{2} \frac{d x}{\mu} \mid+c \varepsilon^{1+\frac{2 \delta}{3}} \\
\leq & \leq c|\mu-\bar{\mu}|+c \varepsilon \leq\left(\tilde{c}_{0} \delta+c\right) \varepsilon^{\frac{1}{2}+\delta} \ln \left(\frac{1}{\varepsilon}\right) .
\end{aligned}
$$

Integrating, we obtain the desired estimate for $|\phi-\bar{\phi}|$. Similarly we obtain the estimate for $|a-\bar{a}|$.

Let $0<\rho \ll 1$. Suppose $\varepsilon$ is small enough such that $\varepsilon^{\rho} \ln \left(\frac{1}{\varepsilon}\right)^{2} \leq 1$. Then we have that

$$
|\phi-\bar{\phi}| \leq \tilde{c}_{0} \delta \varepsilon^{2 \delta} \ln \left(\frac{1}{\varepsilon}\right)^{2} \leq c \varepsilon^{2 \delta-\rho}
$$


If $2 \delta-\rho \geq \frac{1}{2}+\frac{\delta}{3}$, which is equivalent to $\delta \geq \frac{3}{10}+\frac{3}{5} \rho>\frac{3}{10}$, then one can easily see that $|\phi-\bar{\phi}| \leq \varepsilon^{\frac{1}{2}+\frac{\delta}{3}}$. This together with the approximations for $a, \alpha, \mu$ in equations (1.10) yields

$$
\left\|\alpha(t) e^{i \phi(t)} \mu(t) \eta(\mu(t)(x-a(t)))-\bar{\alpha}(t) e^{i \bar{\phi}(t)} \bar{\mu}(t) \eta(\bar{\mu}(t)(x-\bar{a}(t)))\right\|_{H_{+}^{\frac{1}{2}}} \leq c \varepsilon^{\frac{1}{2}+\frac{\delta}{3}} .
$$

Thus, if $\delta \geq \frac{3}{10}+\frac{3}{5} \rho>\frac{3}{10}$, we have that

$$
\left\|u(t)-\bar{\alpha}(t) e^{i \bar{\phi}(t)} \bar{\mu}(t) \eta(\bar{\mu}(t)(x-\bar{a}(t)))\right\|_{H_{+}^{\frac{1}{2}}} \leq c \varepsilon^{\frac{1}{2}+\frac{\delta}{3}},
$$

for times $0 \leq t \leq \frac{\delta}{6 \ln c_{0}} \cdot \frac{1}{\varepsilon^{\frac{1}{2}-\delta}} \ln \left(\frac{1}{\varepsilon}\right)$.

Acknowledgments: The author is grateful to her Ph.D. advisor Prof. Patrick Gérard for suggesting this problem to her and for helpful comments on the paper.

\section{References}

[1] J. C. Bronski, R. L. Jerrard, Soliton dynamics in a potential, Mathematical Research Letters 7 (2000), 329-42.

[2] J. Fröhlich, S. Gustafson, B. L. G. Jonsson, I. M. Sigal, Solitary wave dynamics in an external potential, Communications in Mathematical Physics 250 (2004), 613-42.

[3] J. Fröhlich, S. Gustafson, B. L. G. Jonsson, I. M. Sigal, Long time motion of NLS solitary waves in a confining potential, Annales Henri Poincar 7 (2006), 621-660.

[4] J. Fröhlich, T.-P. Tsai, H.-T. Yau, On the point-particle (Newtonian) limit of the nonlinear Hartree equation, Communications in Mathematical Physics 225 (2002), 223-74.

[5] P. Gérard, S. Grellier, The cubic Szegö equation, Annales Scientifiques de l'Ecole Normale Supérieure, Paris, $4^{e}$ série, t. 43, (2010), 761-810.

[6] P. Gérard, S. Grellier, L'équation de Szegö cubique, Séminaire X EDP, 20 octobre 2008, École Polytechnique, Palaiseau, http://sedp.cedram.org/cedram-bin/article/SEDP_2008-2009_-A2_0.pdf

[7] J. Holmer, M. Zworski, Slow soliton interaction with delta impurities, J. Mod. Dyn. 1 (2007), no. 4, 689-718.

[8] J. Holmer, M. Zworski, Soliton interaction with slowly varying potentials, Int. Math. Res. Not., (2008), no. 10, Art. ID rnn026, 36 pp.

[9] J. Holmer, G. Perelman, M. Zworski, Effective dynamics of double solitons for perturbed $m K d V$, preprint arXiv:0912.5122v2.

[10] S. Keraani, Semiclassical limit of a class of Schrdinger equations with potential, Comm. Partial Differential Equations 27 (2002), no. 3-4, 693704.

[11] S. Keraani, Semiclassical limit for nonlinear Schrdinger equation with potential. II, Asymptot. Anal. 47 (2006), no. 3-4, 171-186.

[12] N.K. Nikolskii, Operators, Functions and Systems: An Easy Reading, Vol.I: Hardy, Hankel, and Toeplitz, Mathematical Surveys and Monographs, vol.92, AMS, (2002).

[13] O. Pocovnicu, Traveling waves for the cubic Szegö equation on the real line, to appear in Analysis and PDE.

Laboratoire de Mathématiques d'Orsay, Université Paris-Sud (XI), 91405, Orsay Cedex, France

E-mail address: oana.pocovnicu@math.u-psud.fr 